Inflammatory bowel disease (IBD). A chronic inflammatory disorder of the gastrointestinal tract with a complex aetiology, including genetic, environmental, microbial and host immune factors. Two major IBD conditions are Crohn's disease (which may affect anywhere in the digestive tract) and ulcerative colitis (which affects only the colon).
${ }^{1}$ Department of Basic and Translational Sciences, Penn Dental Medicine, University of Pennsylvania, Philadelphia, $P A, U S A$.

${ }^{2}$ Institute for Clinical Chemistry and Laboratory Medicine, Faculty of Medicine, Technische Universität Dresden, Dresden, Germany.

凶e-mail:geoh@upenn.edu

https://doi.org/10.1038 s41577-020-00488-6

\title{
Local and systemic mechanisms linking periodontal disease and inflammatory comorbidities
}

George Hajishengallis (1) ${ }^{1 凶}$ and Triantafyllos Chavakis (1D) ${ }^{2}$

Abstract | Periodontitis, a major inflammatory disease of the oral mucosa, is epidemiologically associated with other chronic inflammation-driven disorders, including cardio-metabolic, neurodegenerative and autoimmune diseases and cancer. Emerging evidence from interventional studies indicates that local treatment of periodontitis ameliorates surrogate markers of comorbid conditions. The potential causal link between periodontitis and its comorbidities is further strengthened by recent experimental animal studies establishing biologically plausible and clinically consistent mechanisms whereby periodontitis could initiate or aggravate a comorbid condition. This multi-faceted 'mechanistic causality' aspect of the link between periodontitis and comorbidities is the focus of this Review. Understanding how certain extra-oral pathologies are affected by disseminated periodontal pathogens and periodontitis-associated systemic inflammation, including adaptation of bone marrow haematopoietic progenitors, may provide new therapeutic options to reduce the risk of periodontitis-associated comorbidities.

Periodontitis is a chronic inflammatory disease that progressively affects the integrity of the tissues supporting the teeth (BOX 1) and is associated epidemiologically with several chronic disorders, such as cardiovascular disease, type 2 diabetes mellitus (T2DM), rheumatoid arthritis, inflammatory bowel disease (IBD), Alzheimer disease, nonalcoholic fatty liver disease and certain cancers ${ }^{1-5}$ (FIG. 1). Whereas an imbalanced interaction between the periodontal microbiome/microbiota and the inflammatory response of the host can readily explain local tissue destruction in periodontitis (BOX 1), it has been largely uncertain whether and how this imbalanced relationship can causally link periodontitis and extra-oral comorbidities. From a medical and therapeutic standpoint, it is imperative to understand whether the link between periodontitis and associated comorbidities is merely a correlative one or orchestrated by causal mechanistic interactions.

Recent animal model studies have demonstrated biologically plausible mechanisms whereby periodontitis may increase susceptibility to comorbidities ${ }^{6-16}$. Here, we discuss how periodontal bacteria or locally activated lymphocytes may disseminate to extra-oral tissues, where they may cause inflammatory and functional complications (for example, endothelial cell dysfunction, bone marrow alterations, gut dysbiosis and immune suppression) that aggravate or in certain cases even initiate comorbid pathologies ${ }^{6-16}$. Moreover, recent advances in understanding the epigenetic basis of innate immune memory ${ }^{17}$ (BOX 2) suggest that the inflammatory adaptation of haematopoietic progenitors in the bone marrow may connect multiple chronic inflammatory diseases ${ }^{18}$. The mechanisms linking periodontitis to extra-oral comorbidities are consistent with clinical observations associating periodontitis with bacteraemias, low-grade systemic inflammation, increased myelopoietic activity and the ability of local periodontal treatment to attenuate systemic inflammatory markers and improve comorbid disease activity (surrogate markers) ${ }^{1,19-22}$. Conversely, systemic diseases such as T2DM can promote susceptibility to periodontitis by increasing the inflammatory burden on the periodontium or by modulating the periodontal microbiome ${ }^{1,23,24}$. Therefore, although a reciprocal relationship likely exists between periodontitis and comorbidities, this Review focuses primarily on how periodontitis influences comorbid conditions.

The Review aims to substantiate that the mouthbody link is not simply a consequence of common risk factors but is driven, in substantial part, by multiple microorganism-induced immunological mechanisms that converge to increase the susceptibility of patients with periodontitis to non-communicable chronic inflammatory diseases. Although it is not possible to cover all potential comorbid associations ${ }^{25}$, emphasis is given to epidemiologically strong associations that are also supported mechanistically by clinically relevant experimental evidence. Achieving a comprehensive mechanistic understanding of periodontal 
Microbiome/microbiota

Microbiota is a diverse microbial community that exists within a defined anatomical niche (for example, an environmentally exposed surface of a mammalian organism). The term microbiome represents the microbial community, its combined genetic material and its collective functions.

\section{Dysbiosis}

An imbalanced interaction, between bacteria in a community and/or between the microbial community and the host immune system, which has detrimental effects on the host (as in periodontitis or inflammatory bowel disease). The microbial imbalance derives from changes in the abundance and/or influence of individual species relative to their abundance or influence in health.

Periodontal treatment A procedure that involves mechanical debridement (scaling and root planing) to remove the microbial biofilm (dental plaque) and calculus (tartar) from the tooth surfaces and beneath the gingiva to enable inflammation resolution, and to smooth the root surfaces to deter further biofilm/calculus build-up. Advanced periodontitis may additionally require periodontal surgery, such as pocket reduction surgery, to reduce the depth of the pockets between the teeth and the surrounding gingiva.

\section{Inflammophilic}

From inflammation and the Greek suffix philic indicating fondness. A property of bacteria that thrive under inflammatory conditions by utilizing products derived from the inflammatory breakdown of tissues as nutritional substrates.

Periodontal pockets The physiological narrow space between the tooth root and the free gingiva is known as subgingival crevice; during periodontitis progression, however, this crevice deepens into a periodontal pocket, which is a pathognomonic feature of the disease.

\section{Box 1 | Overview of periodontitis and its animal models}

Periodontitis became prevalent during the transition from hunter-gatherer to Neolithic farming societies ( $\sim 10,000$ years ago), coinciding with dietary changes and a compositional shift of the oral microbiota to a community enriched in periodontitis-associated bacteria, including Porphyromonas gingivalis ${ }^{149}$. This oral disease remains a major public health and socioeconomic burden and afflicts, in its severe form, $\sim 10 \%$ of adults ${ }^{150}$. Periodontitis is an exemplar of an imbalanced interaction between the local microbiome and the inflammatory response of the host (dysbiosis, see the figure). Although induced ostensibly to control the microbial challenge, the inflammatory response is ineffective and poorly controlled in susceptible individuals, leading to inflammatory destruction of the periodontium, a collective term for the tissues that surround and support the dentition (gingiva, periodontal ligament and alveolar bone) ${ }^{87}$. If untreated, the disease can lead to tooth loss, compromising mastication, aesthetics and quality of life $\mathrm{e}^{150}$. Periodontitis can be simulated in animals by placing silk ligatures around posterior teeth (ligature-induced periodontitis); this generates a biofilm-retentive milieu leading to selective expansion of indigenous pathobionts and induction of gingival inflammation and bone loss ${ }^{151,152}$. Another widely used model involves oral gavage with human periodontal pathogens, most often using P. gingivalis. This pathogen colonizes the periodontium and causes quantitative and compositional alterations to the commensal microbiota, thereby instigating inflammatory bone loss ${ }^{153,154}$. Bone loss is mediated - in both mice and humans - largely by T helper $17\left(\mathrm{~T}_{H} 17\right)$ cell-derived IL-17, which induces expression of receptor activator of NF- $\mathrm{kB}$ ligand (RANKL) in osteoblasts, leading to osteoclast activation and bone resorption ${ }^{60,151}$. The pathogenic process is not linear but instead represents a positive-feedback loop between the dysbiotic microbiome and the host inflammatory response. Indeed, while dysbiosis enhances destructive inflammation (largely through activation of complement and Toll-like receptors (TLRs)), inflammation generates a nutritionally favourable environment for selective expansion of periodontitis-associated organisms, termed inflammophilic pathobionts. This reciprocal reinforcement between dysbiosis and inflammation perpetuates a vicious cycle that drives periodontal disease pathogenesis ${ }^{87}$.

comorbidities may not only support the notion that periodontitis is a modifiable risk factor for life-threatening disorders but may also lead to new overarching concepts explaining comorbid inflammatory pathologies, thereby revealing new therapeutic options applicable to multiple inflammatory disorders.

\section{Periodontitis and systemic inflammation}

Although periodontitis shares inflammatory effector mechanisms, as well as genetic and acquired risk factors, with many comorbid conditions, an independent association still remains between periodontitis and comorbidities even after adjustment for confounders ${ }^{1,26,27}$. A possible factor contributing to this independent association is that periodontitis can cause low-grade systemic inflammation, which may influence the development of comorbidities. In comparison with healthy controls, patients with severe periodontitis have elevated levels of pro-inflammatory mediators (such as IL-1, IL-6, $\mathrm{C}$-reactive protein (CRP) and fibrinogen) and increased neutrophil numbers in the blood ${ }^{5,28-30}$. A prospective study involving 11,869 individuals showed that failure to maintain oral hygiene was associated with elevated low-grade systemic inflammation and enhanced risk of cardiovascular disease (CVD) ${ }^{31}$. Conversely, successful local periodontal treatment attenuates systemic inflammatory markers ${ }^{5,19,22,29,30,32,33}$.
Periodontitis-associated systemic inflammation likely results from haematogenous dissemination of periodontal bacteria or spillover of inflammatory mediators from periodontal tissues to the bloodstream. In this regard, the ulcerated epithelium of the periodontal pockets covers a surface area of $8-20 \mathrm{~cm}^{2}$ and may allow bacteria and their products (for example, lipopolysaccharide (LPS) or proteases) to reach the circulation, causing bacteraemias (FIG. 2), which are documented in patients with periodontitis ${ }^{5,21}$. Inflammation in extra-oral sites can be induced also through oro-pharyngeal or oro-digestive translocation of periodontal bacteria; the former is associated with aspiration pneumonia ${ }^{34}$, whereas the latter is associated with intestinal dysbiosis and gut-mediated systemic inflammation ${ }^{6,8,35,36}$. The elevated systemic inflammation associated with periodontitis may have multiple systemic complications as outlined below.

\section{Immunometabolic alterations}

Evolutionarily, host survival was strongly dependent on two traits: a powerful immune response to fight off infections and an effective calorie-storage system to withstand starvation. Although the haematopoietic/ immune system and liver and adipose tissue represent distinct tissues and organs in higher organisms, their functions in primitive organisms are performed by a single organ (for example, the fat body in Drosophila) ${ }^{37}$. 
Nonalcoholic fatty liver disease/nonalcoholic steatohepatitis

(NAFLD/NASH). NAFLD

is a condition associated with obesity and metabolic syndrome and involves excessive fat accumulation (steatosis) in the liver in the absence of significant alcoho consumption. NAFLD may progress to NASH, where fat accumulation in the liver is accompanied by inflammation, leading to fibrosis and ultimately cirrhosis (advanced scarring/fibrosis) and end-stage liver failure.

$\mathrm{HbA}_{1 c}$

$\mathrm{HbA}_{1}$, or glycated

haemoglobin, represents a form of haemoglobin that has glucose attached to it via a non-enzymatic reaction called glycation. The concentration of $\mathrm{HbA}_{1 \mathrm{c}}$ in blood reflects the average levels of blood glucose of the previous 3-4 months. Therefore, $\mathrm{HbA}_{\text {, }}$ is used not only in the diagnosis of diabetes but, importantly, as a measure for monitoring glycaemic control in patients with diabetes
Not surprisingly, the host immune response is tightly intertwined with metabolism, and dysfunction of this integrated system may lead to chronic metabolicinflammatory disorders, such as obesity and related pathologies, including the metabolic syndrome, T2DM, nonalcoholic fatty liver disease/nonalcoholic steatohepatitis (NAFLD/NASH) and $\mathrm{CVD}^{38}$. Chronic low-grade inflammation is a unifying feature and contributor to these disorders ${ }^{38}$; at least in principle, therefore, periodontitis may affect metabolic-inflammatory conditions by increasing the systemic inflammatory burden $^{5,19,22,28-30,32,33}$. In this regard, periodontitis is associated with elevated serum levels of low-density lipoprotein (LDL) and triglycerides and reduced levels of high-density lipoprotein (HDL) ${ }^{39}$. The association of periodontitis, obesity, dyslipidaemia, T2DM, NAFLD/ NASH and CVD (reviewed in REFS ${ }^{4,39-41}$ ) might be attributed, in part, to causal relationships, as suggested by clinical interventions.
A recent study showed that effective periodontal treatment reduces systemic inflammatory markers and favourably influences the metabolic status of patients with T2DM (who showed reduction of plasma glucose concentrations and $\mathrm{HbA}_{1 \mathrm{c}}$ ), as well as leading to improvements in vascular and kidney functions ${ }^{19}$. In patients with periodontitis who do not have diabetes, periodontal treatment improves endothelial function, as assessed by flow-mediated dilatation ${ }^{42}$. A longitudinal improvement in periodontal health is linked to attenuated progression of intima-medial thickness of the carotid artery, a marker of atherosclerosis ${ }^{43}$. Periodontal treatment of patients with periodontitis who are hyperlipidaemic reduces LDL and triglyceride levels and increases HDL levels ${ }^{39}$. Periodontal treatment of patients with NAFLD/NASH decreases the serum levels of hepatic-parenchymal damage markers, aspartate aminotransferase and alanine aminotransaminase ${ }^{44}$, and in patients with cirrhosis, periodontal treatment reduces local and systemic

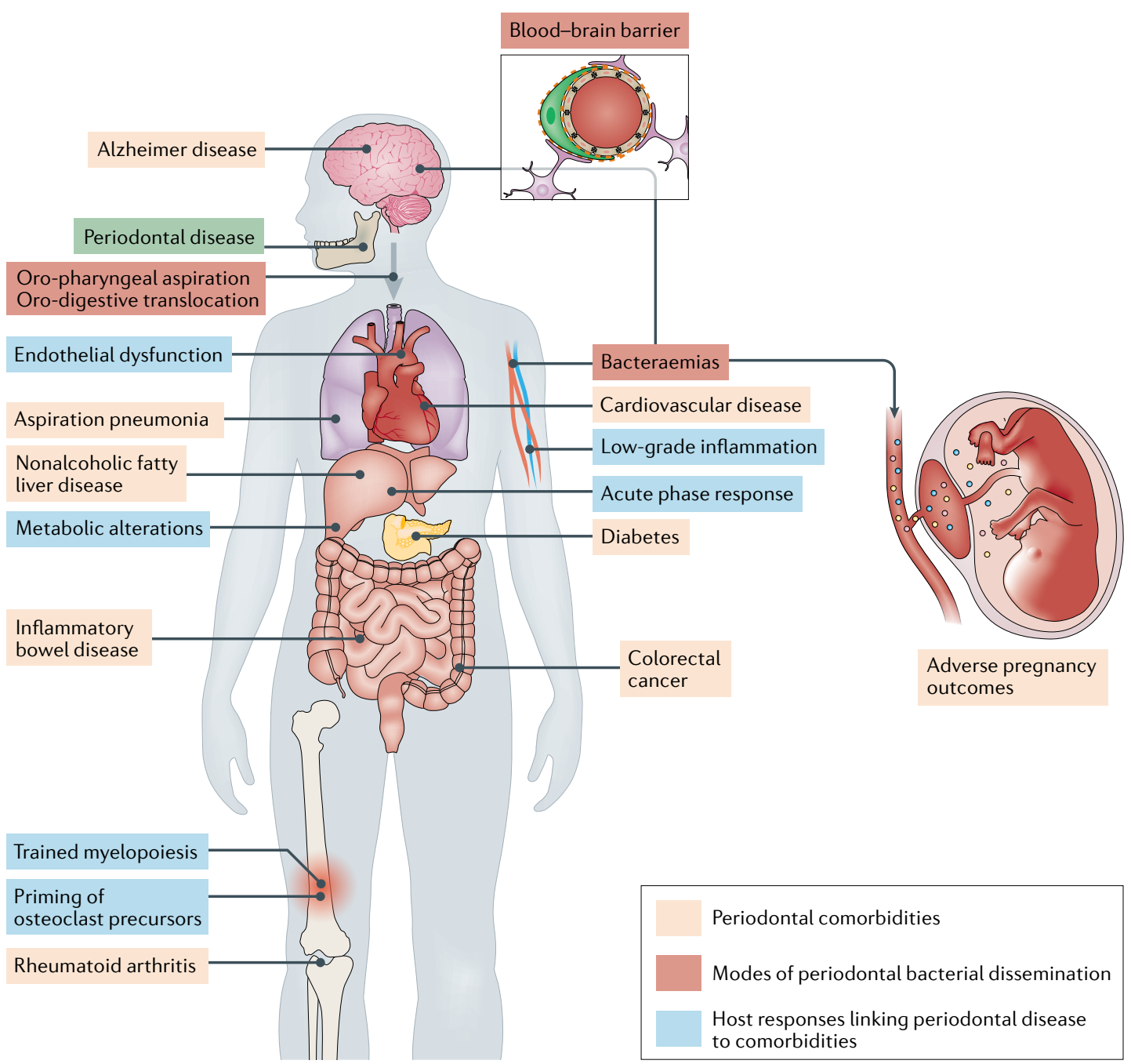

Fig. 1 | Periodontal disease and associated inflammatory comorbidities. On the basis of epidemiological, clinical intervention and animal model-based studies, periodontitis has been linked with a number of comorbid conditions, such as those indicated. Mechanistically, periodontitis is associated with bacteraemias and systemic inflammation, which can induce acute-phase responses as well as metabolic and inflammatory alterations in the liver and bone marrow, activities that can influence comorbid conditions. Moreover, periodontal bacteria can disseminate by different routes - haematogenous, oro-pharyngeal and oro-digestive - to reach extra-oral sites where they can cause or exacerbate inflammatory pathologies. 


\section{Box 2 | Trained immunity and maladaptive inflammation}

Immunological memory is the ability to recall past pathogen encounters and initiate a faster and stronger immune response upon re-challenge; this crucial feature of immunity has been hitherto considered an exclusive prerogative of adaptive immunity ${ }^{17,155}$. However, recent studies have shown that earlier microbial or inflammatory challenges (for example, those triggered by damage-associated molecular pattern (DAMP) molecules) imprint a form of memory also in innate immune cells that enables them, upon returning to a non-activated state, to respond more robustly to secondary heterologous challenges. This state of enhanced immune responsiveness is termed trained immunity and is shaped by long-term epigenetic and metabolic alterations, as opposed to permanent genetic changes (gene recombination and somatic hypermutation) that characterize classical adaptive immunological memory (see the figure ${ }^{17,155}$. Innate immune memory lacks the specificity of its adaptive counterpart and can confer broad-based protection against future infections with the original or even unrelated pathogens. A trained phenotype (typified by epigenetic and transcriptional alterations and heightened immune preparedness) was initially documented in mature myeloid cells; however, the long-lasting (several months) consequences of trained immunity could not be reconciled with the relatively short lifespan (a few days) of circulating myeloid cells. This paradox was resolved when trained immunity was shown to be initiated in bone marrow haematopoietic stem and progenitor cells (HSPCs). Inflammation-induced metabolic, epigenetic and transcriptomic adaptations in HSPCs instruct them to undergo enhanced proliferation and myeloid-biased differentiation, generating trained or hyper-reactive myeloid cell populations ${ }^{68,156}$. Although trained immunity is an evolutionarily conserved function that promotes host survival upon re-infection ${ }^{17,155}$, it may boost chronic inflammation in a detrimental manner, as exemplified in cardiometabolic diseases, in which increased bone marrow haematopoietic activity drives persistent leukocytosis and contributes to their chronicity ${ }^{157-160}$. HSPCs that are epigenetically trained (by earlier infection or injury) to elicit a heightened myelopoietic response may also exacerbate other inflammatory or autoimmune conditions with myeloid cell involvement.

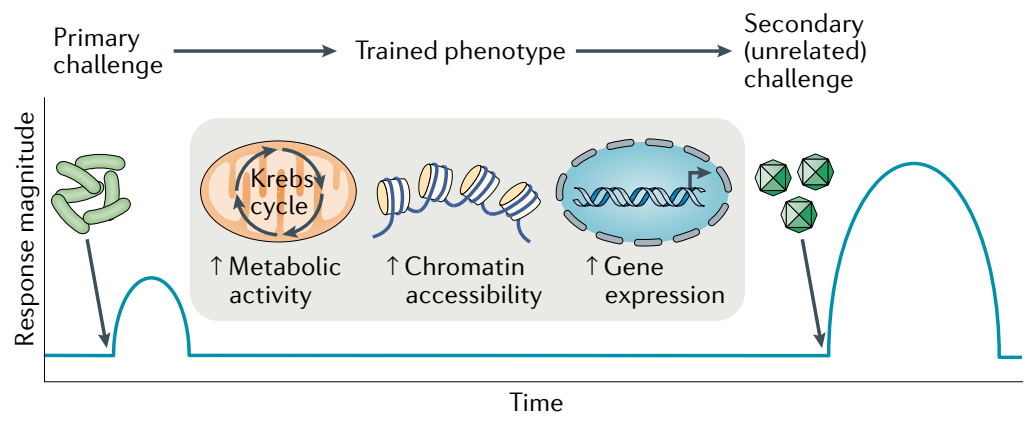

Atheromatous plaque Fat accumulation in the intima (inner lining) of arteries that results in fibrous thickening or calcification of the arteries, restriction of blood flow and an enhanced risk of thrombotic occlusion. inflammation and endotoxaemia, as well as improving cognitive function and quality of life in those with prior hepatic encephalopathy ${ }^{22}$. Consistently, a cohort study of 6,165 individuals, without baseline liver disease, correlated periodontitis with incident liver disease independently of confounders ${ }^{45}$.

The notion that periodontitis causes systemic metabolic complications is supported by studies in rodents or lagomorphs subjected to procedures used as experimental models of periodontitis, such as ligature-induced periodontitis (LIP) or oral gavage with human periodontal pathogens (BOX 1). A few examples are given here. Similarly to what is seen in human periodontitis, experimental animal periodontitis causes increased serum levels of acute phase proteins (such as CRP and serum amyloid A) and inflammatory cytokines (for example, IL-1 $\beta$ and IL-6) (6,46-49 $^{6}$ and is thus an appropriate model to study the periodontitis connection with systemic comorbidities. LIP in rats leads to vascular inflammation and endothelial dysfunction that is accompanied by increased neutrophil numbers and increased serum levels of CRP, IL-6 and LDL cholesterol ${ }^{46}$. Vascular inflammation can likely be attributed, in part, to periodontitis-induced activation of circulating monocytes and their enhanced adhesion to aortic endothelial cells via nuclear factor- $\kappa \mathrm{B}$ (NF- $\kappa \mathrm{B})$ p 65 nuclear translocation and upregulation of VCAM1 in the latter ${ }^{50}$. LIP in rabbits fed a high-cholesterol diet causes formation of more aortic atheromatous plaque than occurs in periodontally healthy controls under the same diet, and local treatment of periodontitis with a pro-resolving agent (resolvin E1) attenuates LIP-induced vascular inflammation and atherogenesis ${ }^{51}$.

In mice fed a high-fat diet (HFD), oral gavage with a combination of three human periodontal pathogens (Porphyromonas gingivalis, Fusobacterium nucleatum and Prevotella intermedia) not only induced local inflammatory bone loss but also appeared to aggravate HFD-induced glucose intolerance and insulin resistance and increased the ratio of fat to lean tissue mass ${ }^{9}$. These findings were reproduced in HFD-fed mice subjected to continuous low-rate infusion of $P$. gingivalis LPS, mimicking chronic low-grade systemic inflammation'. Additionally, periodontal pathogens might potentially aggravate insulin resistance through their metabolic activities. In a model of $P$. gingivalis-induced periodontitis in HFD-fed mice, infection with the wild-type organism, but not with a branched-chain amino acid (BCAA) aminotransferase-deficient mutant, resulted in increased serum levels of BCAAs (such as Leu, Ile and $\mathrm{Val}$ ) and insulin resistance, as compared with uninfected HFD-fed mice ${ }^{52}$. This is consistent with the notion that elevated circulating BCAAs contribute to increased risk of T2DM ${ }^{53}$. Periodontitis-associated insulin resistance and the resulting hyperglycaemia may also induce formation of advanced glycation end products (AGEs), leading to enhanced activation of the pro-inflammatory receptor for AGE (RAGE), which is involved in several metabolic disorders, including vascular inflammation ${ }^{54}$ and atherosclerosis ${ }^{55}$, as well as in experimental periodontitis in diabetic mice ${ }^{56}$.

In an HFD-induced NAFLD model in mice, intravenous administration of $P$. gingivalis (but not of oral streptococcal species) significantly increased body and liver weight and elevated alanine aminotransaminase and liver triglyceride concentrations, compared with HFD controls ${ }^{44}$. Similarly, P. gingivalis infection of mice subjected to NASH appeared to promote steatosis and hepatic stellate cell activation, thereby causing more severe fibrosis (relative to uninfected NASH controls) associated with altered fatty acid metabolism in the liver $^{57,58}$. LIP in rats resulted in steatosis, likely attributed to increased oxidative stress and lipid peroxidation, as suggested by lower glutathione and higher malondialdehyde concentrations than those of the unligated controls ${ }^{59}$.

Although systemic inflammation induced by bloodborne periodontal pathogens may explain, to a significant extent, the above-discussed metabolic alterations, direct action of translocated pathogens in the liver cannot be ruled out. In this regard, in LIP-subjected mice, viable oral bacteria could be recovered from the liver and spleen until the periodontitis-affected teeth were 
Emergency myelopoiesis A tightly regulated process initiated in response to systemic infection for de novo generation of mature myeloid cells, resulting from accelerated proliferation and enhanced myeloid differentiation of progenitors in the bone marrow. The goal is to meet the increased demand for neutrophils and other phagocytes that are consumed in large quantities during systemic infections.

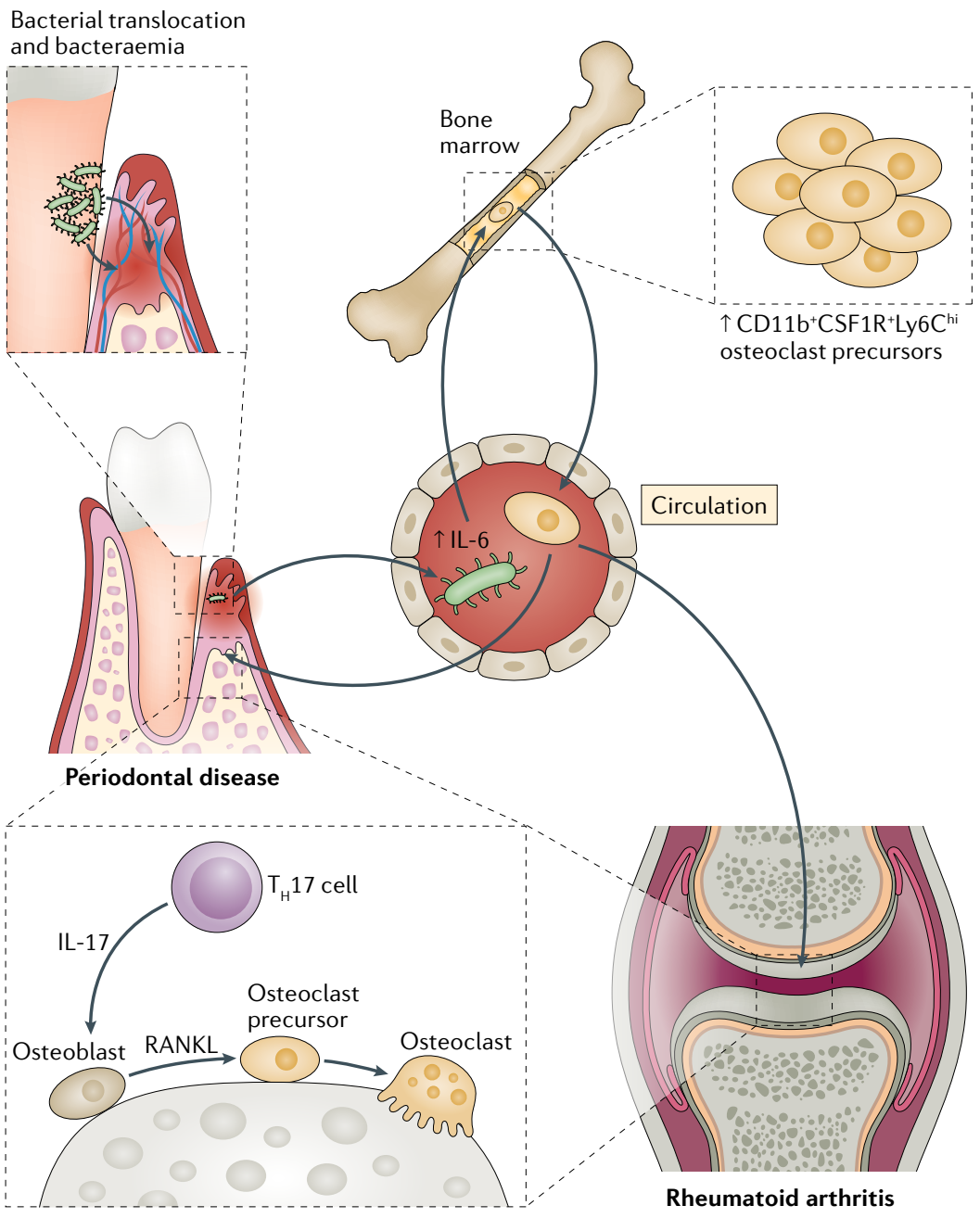

Fig. 2 | Periodontal bacterial translocation leading to bacteraemia and alterations in the bone marrow that promote osteoclastogenesis in different sites. Periodontal bacteria may translocate through the ulcerated epithelium of the periodontal pockets into the circulation, causing bacteraemia and systemic inflammation. Blood-borne Porphyromonas gingivalis causes an increase in the serum levels of IL-6, which in turn induces the expansion of an osteoclast precursor population (defined as $\mathrm{CD}_{11} \mathrm{~b}^{+} \mathrm{CSF} 1 \mathrm{R}^{+} \mathrm{Ly}_{6} \mathrm{C}^{\mathrm{hi}}$ ) in the bone marrow. This osteoclast precursor population displays enhanced osteoclastogenic lineage bias and populates various bone resorption sites, where it can differentiate into mature osteoclasts, in response to locally produced receptor activator of NF-kB ligand (RANKL) (for example, produced by osteoblasts stimulated by Thelper 17 ( $\left.\mathrm{T}_{H} 17\right)$ cell-derived IL-17). This concept may be a mechanism whereby the bone marrow might link periodontitis to other bone loss disorders, such as rheumatoid arthritis. alterations to the gut microbiota and aggravated glucose intolerance, insulin resistance and liver steatosis ${ }^{61}$.

\section{Alterations in the bone marrow}

The bone marrow is the primary site of haematopoiesis where all mature blood cell types arise from a common ancestor, the haematopoietic stem cell (HSC) ${ }^{62}$. To optimally perform its function, the bone marrow needs to respond swiftly to peripheral challenges such as infection or injury. For instance, in emergency myelopoiesis, HSCs respond rapidly to systemic infection with increased proliferation and myeloid differentiation leading to enhanced myelopoiesis ${ }^{63}$. In this regard, systemic infections or inflammation can be sensed in the bone marrow by haematopoietic stem and progenitor cells (HSPCs) via Toll-like receptors (TLRs) or receptors for inflammatory cytokines, thereby initiating the process for increased production of cells that fight infections, such as neutrophils and monocytes ${ }^{18}$.

Through further differentiation via macrophage colony-stimulating factor (M-CSF) and receptor activator of NF- $\kappa B$ ligand (RANKL), the monocytic lineage is the precursor of osteoclasts, which are giant multinucleated bone-resorbing cells ${ }^{64}$. Although osteoclast precursors (OCPs) are generated in the bone marrow, they can circulate in the blood and enter sites of active bone resorption, where they can differentiate to osteoclasts. Sustained systemic release of P. gingivalis, via a micro-osmotic pump, to simulate periodontitis-associated bacteraemia in mice, resulted in expansion of an OCP population (defined as $\left.\mathrm{CD} 11 \mathrm{~b}^{+} \mathrm{CSF} 1 \mathrm{R}^{+} \mathrm{Ly} 6 \mathrm{C}^{\mathrm{hi}}\right)$ with enhanced osteoclastogenic lineage bias (relative to the counterpart population from uninfected controls $)^{16}$. This OCP expansion in the bone marrow was dependent on IL- 6 , the serum levels of which were increased in P. gingivalis-infected mice $^{16}$ (FIG. 2). In vivo adoptive transfer and tracking experiments showed that the P. gingivalis-induced OCPs could populate bone resorption sites and differentiate into mature osteoclasts ${ }^{16}$. Consistently, compared with those from healthy controls, peripheral blood mononuclear cells isolated from the blood of patients with periodontitis are more poised to become osteoclasts, as they undergo enhanced RANKL-induced osteoclast differentiation into mature osteoclasts ${ }^{65}$. Thus, periodontal bacteria-induced systemic IL- 6 drives the expansion of OCPs that traffic to sites of bone resorption to boost osteoclastogenesis in response to locally produced RANKL, suggesting that changes in the bone marrow may link periodontitis to other bone loss disorders, such as rheumatoid arthritis (FIC. 2).

As alluded to above, HSPCs are more sensitive than traditionally thought to acute infection or chronic inflammation, as they express TLRs (for example, TLR4 for sensing LPS) and receptors for inflammatory cytokines and growth factors (such as IL-1 $\beta$, IL-6 and M-CSF) ${ }^{62,66}$. Such stimuli induce expansion of HSPCs and their skewed differentiation towards the myeloid lineage. Inflammatory adaptation of HSPCs underlies the persistence of trained immunity ${ }^{18}$ (BOX 2) and is regulated by long-term cell-intrinsic (metabolic, transcriptional and epigenetic) mechanisms. IL-1 $\beta$ plays a significant role 


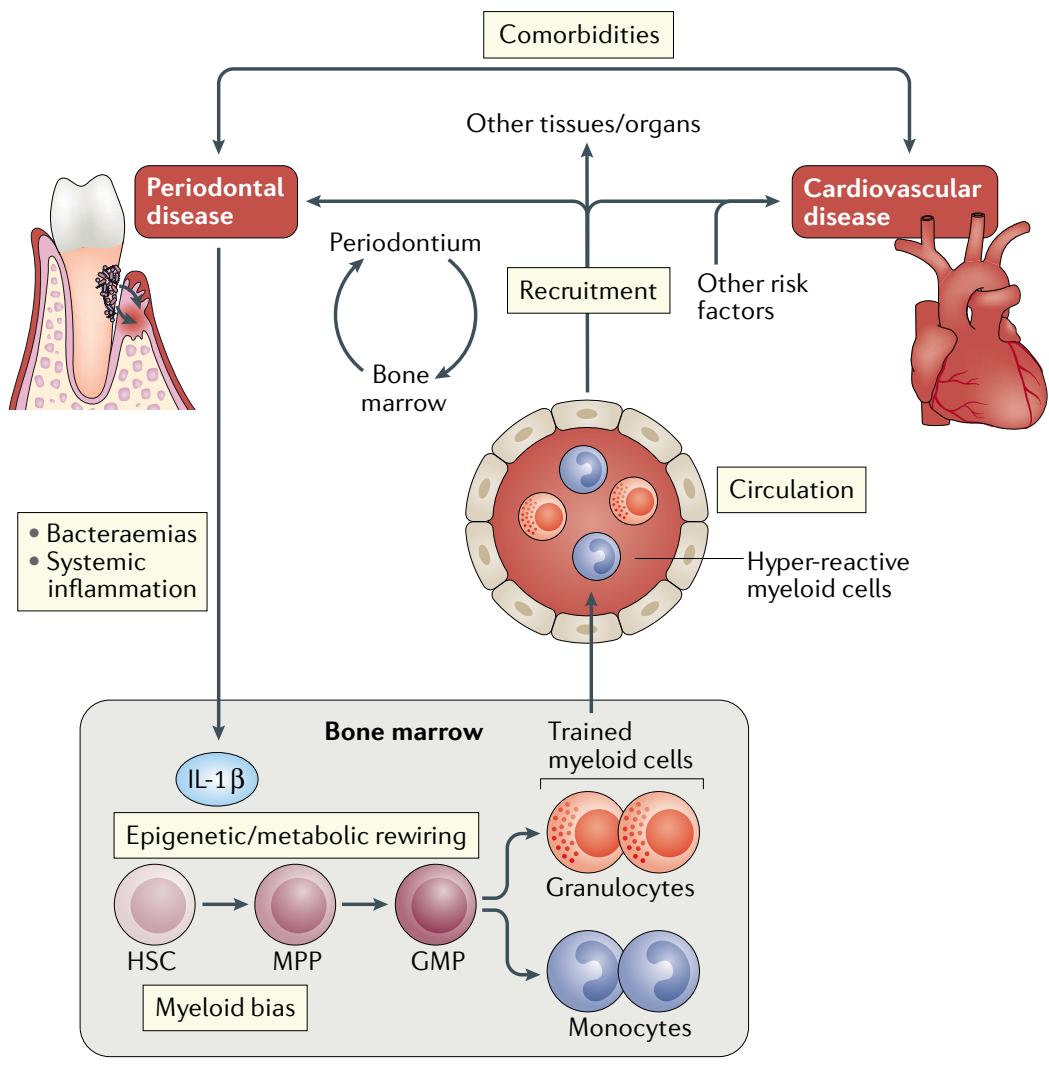

Fig. 3 | Trained myelopoiesis in the bone marrow as a mechanistic basis of inflammatory comorbidities. Periodontitis-associated bacteraemias and systemic inflammation, for example, inflammatory cytokines, such as IL-1 $\beta$, can be sensed in the bone marrow, leading to induction of long-term metabolic and epigenetic rewiring in haematopoietic stem cells (HSCs). These inflammation-adapted HSCs proliferate and preferentially undergo myeloid-biased differentiation, leading to expansion of multipotent progenitors (MPPs) with myeloid potential and granulocyte-macrophage progenitors (GMPs) and ultimately production of 'trained' myeloid cell populations. These hyper-reactive neutrophils or monocytes/macrophages can be recruited to the periodontium and other sites of infection, inflammation or injury, thereby potentially exacerbating periodontitis and promoting inflammatory pathology of comorbid conditions such as cardiovascular disease.

Rheumatoid arthritis A chronic inflammatory autoimmune disease typified by production of autoantibodies to various targets (for example, citrullinated matrix proteins) and induction of inflammation that causes progressive erosion of cartilage and bone in the joints. Its aetiology is complex and includes both genetic and environmental factors. in the induction of trained immunity in humans and mice $^{67,68}$ (FIG. 3). In the bone marrow, this cytokine acts on HSPCs and promotes, likely via changes in their cellular metabolism, a myeloid-differentiation bias, which is a hallmark of HSPCs in trained immunity ${ }^{18,68,69}$.

As a cause of systemic inflammation, periodontitis might, at least in principle, trigger inflammatory adaptation of HSPCs and trained immunity in the bone marrow. This notion is backed by a recent clinical imaging study using ${ }^{18} \mathrm{~F}$-fluorodeoxyglucose positron emission tomography-computed tomography $\left({ }^{18} \mathrm{~F}-\mathrm{FDG}\right.$-PET-CT). This study showed a correlation between metabolic activity within the periodontium (surrogate marker of periodontal inflammation) and haematopoietic tissue activity (reflecting progenitor cell activity and stimulated myelopoiesis), as well as with arterial inflammation ${ }^{20}$. An earlier ${ }^{18} \mathrm{~F}$-FDG-PET-CT study indicated a correlation between periodontal inflammation and/or metabolic activity and histologically confirmed carotid plaque inflammation ${ }^{70}$. Together, these findings suggest that increased haematopoietic activity may account, at least in part, for the association between periodontal and cardiovascular inflammation.

Whether periodontitis can indeed influence haematopoietic tissue activity and trained immunity in the bone marrow has yet to be addressed experimentally. However, certain clinical findings may be consistent with this notion. Upon ex vivo activation by LPS or whole bacteria, peripheral blood neutrophils or monocytes from patients with periodontitis respond with higher pro-inflammatory cytokine production than the same cell types from healthy individuals ${ }^{71,72}$. Interestingly, this hyper-reactivity often remains even after successful periodontal therapy; that is, the higher capacity to produce cytokines seen in myeloid cells from patients versus healthy controls persists after therapy for at least 2 months (the time interval for post-treatment review) ${ }^{71,72}$. These data resemble findings from individuals treated with agents that are associated with induction of trained immunity, such as the Bacillus Calmette-Guérin (BCG) vaccine $^{73,74}$, and could thus be interpreted to mean that the peripheral blood myeloid cells of patients with periodontitis are in a trained state, which enables them to induce a heightened response (compared with cells from healthy controls) to future inflammatory challenges. Given that periodontitis affects haematopoietic tissue activity ${ }^{20}$, this trained state of peripheral myeloid cells might be the result of long-term epigenetic immune memory imprinted in inflammation-adapted HSPCs in the bone marrow ${ }^{17,68}$. Similarly, a subset of patients with rheumatoid arthritis in clinical remission exhibit increased bone marrow and splenic metabolic activity as well as increased arterial wall inflammation together with a promigratory phenotype of circulating monocytes ${ }^{75}$. Thus, being in remission does not necessarily normalize the risk of developing CVD in patients with rheumatoid arthritis. Conversely, cardiometabolic conditions were shown to influence haematopoiesis and trained immunity ${ }^{76-78}$.

These findings are consistent with the concept that an inflammatory disease may affect another disease quantitatively by enhancing myelopoiesis in the bone marrow or qualitatively by driving generation of myeloid cells with enhanced inflammatory potential, even in patients in remission (implying the presence of innate immune memory in HSPCs). Regardless of whether the bone marrow is first affected by periodontitis-induced systemic inflammation or systemic inflammation caused by another condition, such as CVD, the resulting heightened and sustained ('trained') myelopoiesis can have an impact on both conditions (FIG. 3). The production of trained/ hyper-reactive myeloid progeny and their recruitment to inflamed tissues with consequent exacerbation of local pathology could possibly generate a positive-feedback loop between inflammation-adapted haematopoietic progenitor cells in the bone marrow and the inflammatory disorders ${ }^{18}$ (FIG. 3). According to this concept, periodontitis can affect comorbid conditions and vice versa. Consistent with the latter, in a prospective study with 1,784 subjects and an 11-year follow-up, serum fibrinogen and white blood cell count (markers of systemic inflammation) were longitudinally associated with periodontitis severity in a dose-dependent manner ${ }^{79}$. 
Keystone pathogen

A pathogen that exerts a disproportionately large effect on its community relative to its abundance, forming the 'keystone' of the community's structure; for instance, in experimental periodontitis, low-abundance Porphyromonas gingivalis modulates the size and composition of the local microbial community in a manner that promotes dysbiosis.
Peripheral blood neutrophils from patients with periodontitis display increased expression of interferonstimulated genes that correlates with increased plasma IFN $\alpha$ concentrations, relative to healthy individuals ${ }^{80}$. Ex vivo, these neutrophils exhibit a hyper-reactive phenotype in terms of reactive oxygen species (ROS) production when primed with type I interferons (but not LPS $)^{80}$. Whether this type I interferon-associated hyper-reactivity of peripheral blood neutrophils from patients with periodontitis arises from trained granulopoiesis in the bone marrow is uncertain. Interestingly, mice systemically treated with fungally derived $\beta$-glucan, a known inducer of trained immunity, display type I interferon-dependent trained granulopoiesis with transcriptomic and epigenetic rewiring of granulopoietic progenitors in the bone marrow, leading to the generation of neutrophils with an enhanced ROS-dependent tumour-killing phenotype ${ }^{81}$. Thus, trained granulopoiesis

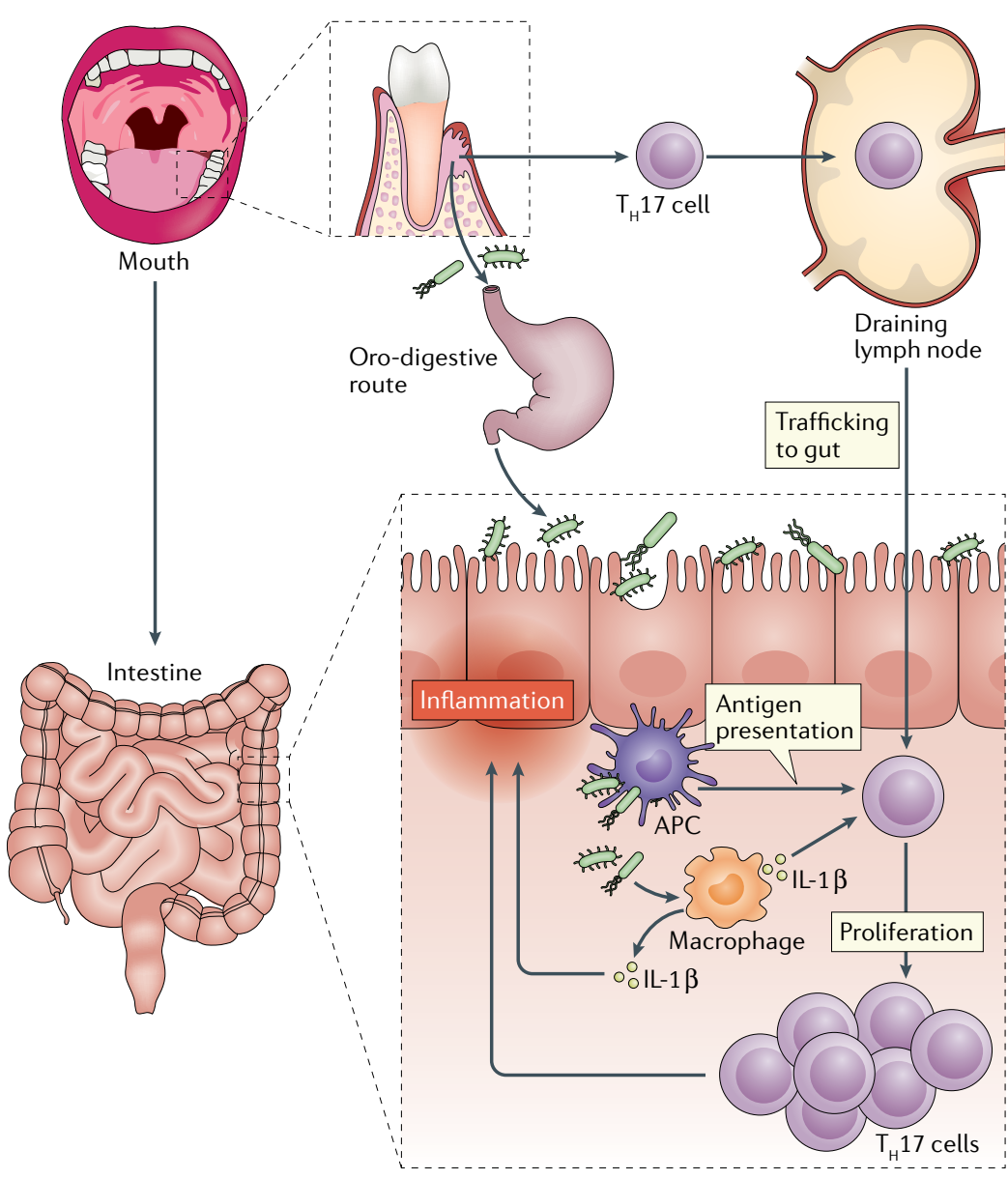

Fig. 4 | Oral-gut axis mechanisms that promote colitis. Periodontitis-associated pathobionts can reach the gut through the oro-digestive route (owing to constant saliva swallowing) and can promote colitis in susceptible hosts, in part through induction of IL-1 $\beta$ by inflammatory macrophages. Oral pathobiont-reactive T cells (enriched in Thelper $17\left(\mathrm{~T}_{\mathrm{H}} 17\right)$ cells), which expand during periodontitis, migrate through the lymphatics to the gut, where they are activated by the ectopically colonized oral pathobionts upon their processing by antigen-presenting cells (APCs). The oral pathobiont-induced elevation of IL-1 $\beta$ contributes to the activation and proliferation of the transmigrated $\mathrm{T}_{H} 17$ cells, which become colitogenic and exacerbate intestinal inflammation. Thus, ectopically colonized oral pathobionts may exacerbate colitis by activating both innate immunity (local inflammatory macrophages) and adaptive immunity (transmigrated $\mathrm{T}_{H} 17$ cells of oral origin). induced by systemic inflammation (due to treatment with purified agonists or perhaps associated with periodontitis or other condition) might lead to the generation of hyper-reactive neutrophils, which, depending on the disease context, can be protective (for example, in tumour immunity) or destructive (for instance, in inflammatory conditions).

\section{Direct effects of periodontal bacteria}

Multiple clinical studies have detected genomic DNA from periodontal bacteria in systemic tissues; however, there is limited evidence for their presence in a viable state $^{82,83}$. Although periodontal bacteria have evolved immune subversive strategies that enable them to thrive in periodontal pockets ${ }^{3}$, circulating periodontal pathogens may be more vulnerable to phagocytic killing in the blood or in systemic tissues. Nevertheless, even if the presence of viable periodontal bacteria in systemic tissues is transient, their effects - for instance, their release of virulence factors, such as toxic proteases ${ }^{11,15}$, or their induction of inflammation - can be important, given the chronicity of periodontitis and the frequency of bacteraemias, which occur not only during professional dental care (for example, debridement of the pathogenic dental biofilm, tooth extractions) but also during routine activities (such as tooth brushing or mastication $)^{21}$. Periodontal bacteria, such as $P$. gingivalis, are also detected (by $16 \mathrm{~S}$ ribosomal DNA sequencing) in blood myeloid-derived dendritic cells (mDCs $)^{84}$. Bacteraemias induced by debridement elevate the $P$. gingivalis content of blood $\mathrm{mDCs}$ and the frequency of the carrier $\mathrm{mDCs}$, which have been shown by immunohistochemistry to colocalize with $P$. gingivalis in atheromatous plaques of patients with periodontitis. As $P$. gingivalis may survive within mDCs in vitro, this mechanism might serve to disseminate the pathogen to remote sites, such as atheromas ${ }^{84}$. Non-haematogenous dissemination of periodontal bacteria has also been described. Swallowed periodontal bacteria may translocate via the oro-digestive route and ectopically colonize the gut (FIG. 4); indeed, periodontal bacteria have been detected in the gut of patients with $\mathrm{IBD}^{8,85,86}$. Interestingly, periodontal bacteria may not necessarily have to relocate from the periodontium to affect pathology in distant sites, such as the joints ${ }^{2,12}$. Below, we consider in more detail the effects of disseminated periodontal bacteria as well as the effects of locally acting periodontal bacteria on distal tissues (TABLE 1).

Endothelial dysfunction. The gingipain proteases of the periodontal keystone pathogen $P$. gingivalis are critical for its capacity to colonize subgingival tooth sites, procure nutrients, subvert immunity and contribute to periodontal tissue destruction ${ }^{2,87}$. Gingipains are important also for the virulence of $P$. gingivalis in extra-oral sites. In a zebrafish larva infection model, $P$. gingivalis induced oedema by increasing vascular permeability, which was attributed to gingipain-dependent degradation of platelet endothelial cell adhesion molecule (PECAM1) and vascular endothelial cadherin (VE-cadherin) disrupting the endothelial barrier ${ }^{15}$ (FIG. 5a). Consistently, human endothelial cells infected by $P$. gingivalis (but not by 
Table 1 | Potential mechanisms of human periodontal bacterial involvement in extra-oral pathologies

\begin{tabular}{|c|c|c|c|c|}
\hline Bacterium & $\begin{array}{l}\text { Effector } \\
\text { molecule }\end{array}$ & Target & Outcome & Refs \\
\hline \multirow[t]{3}{*}{ Porphyromonas gingivalis } & Gingipains & $\begin{array}{l}\text { PECAM1, VE-cadherin, in vascular } \\
\text { endothelial cells }\end{array}$ & $\begin{array}{l}\text { Impairment of endothelial junctional integrity, vascular } \\
\text { damage }\end{array}$ & 15 \\
\hline & PPAD & $\begin{array}{l}\text { Proteins, including host molecules, } \\
\text { such as fibrinogen and } \alpha \text {-enolase }\end{array}$ & $\begin{array}{l}\text { Citrullination of protein autoantigens leading to ACPA } \\
\text { induction }\end{array}$ & $125-127$ \\
\hline & BCAT & Respective $\alpha$-keto acids ${ }^{\mathrm{a}}$ & $\begin{array}{l}\text { Increased plasma levels of BCAAs (Leu, Ile and Val); } \\
\text { insulin resistance }\end{array}$ & 52 \\
\hline $\begin{array}{l}\text { Aggregatibacter } \\
\text { actinomycetemcomitans }\end{array}$ & Leukotoxin A & $\beta 2$ Integrin on neutrophils & $\begin{array}{l}\text { PAD hyperactivation and release of citrullinated } \\
\text { autoantigens through cytolysis, leading to ACPA } \\
\text { induction }\end{array}$ & 12 \\
\hline Klebsiella pneumoniae & $\begin{array}{l}\text { Unspecified } \\
\text { TLR agonists }\end{array}$ & TLRs on colonic epithelial cells and DCs & IL-18 secretion, $T_{H} 1$ cell expansion and gut inflammation & 8 \\
\hline \multirow{2}{*}{$\begin{array}{l}\text { Fusobacterium } \\
\text { nucleatum }\end{array}$} & Fap2 & TIGIT on T cells and NK cells & Tumour immune evasion & 10 \\
\hline & FadA & E-cadherin on CRC cells & $\begin{array}{l}\text { Activation of Wnt- } \beta \text {-catenin signalling and CRC cell } \\
\text { proliferation }\end{array}$ & 112,118 \\
\hline
\end{tabular}

ACPA, anti-citrullinated protein antibody; APOE, apolipoprotein E; BCAA, branched-chain amino acid; BCAT, branched-chain amino acid aminotransferase; CRC, colorectal cancer; DC, dendritic cell; DC-SIGN, DC-specific ICAM3-grabbing non-integrin; NK, natural killer; PAD, peptidyl-arginine deiminase; PECAM1, platelet endothelial cell adhesion molecule; PPAD, Porphyromonas gingivalis peptidyl-arginine deiminase; T 1 cell, T helper 1 cell; TIGIT, T cell

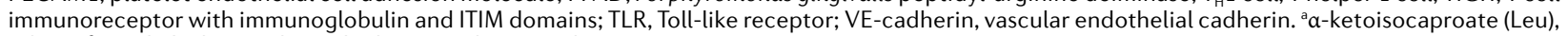
$\alpha$-keto- $\beta$-methylvalerate (lle) and $\alpha$-ketoisovalerate (Val).

Gingipains

A family of Porphyromonas gingivalis-derived trypsin-like cysteine proteases that make a major contribution to its virulence. High-molecular mass arginine-specific gingipain A (HRgpA), arginine-specific gingipain $B(\operatorname{RgpB})$ and lysine-specific gingipain (Kgp) are the members of the gingipain family.

\section{Alzheimer disease}

Progressive neuroinflammatory and neurodegenerative brain disease, characterized by extraneuronal deposition of amyloid- $\beta$ peptides (neuritic plaques) and intraneuronal accumulation of hyperphosphorylated and fragmented tau, a microtubule-associated protein (neurofibrillary tangles) a gingipain-deficient mutant) displayed decreased cell surface expression of PECAM1 and VE-cadherin, disrupted cell-cell junctions and elevated endothelial cell permeability, which was reversed by a specific gingipain inhibitor ${ }^{15}$. Vascular damage and compromised endothelial junctional integrity may potentially trigger platelet aggregation, leukocyte adhesion and induction of pro-inflammatory cytokines, thereby possibly promoting atherosclerosis ${ }^{88}$ (FIG. 5a). It is uncertain whether $P$. gingivalis-induced vascular damage also occurs in humans and contributes to atherosclerotic lesions in susceptible individuals; intriguingly, however, P. gingivalis is the most abundant bacterial species detected in non-diseased vascular tissue of patients with atherosclerosis ${ }^{89}$.

Alzheimer disease. Several epidemiological studies have suggested an association between periodontitis and Alzheimer disease (reviewed in REF. ${ }^{90}$ ). A recent large retrospective cohort study associated clinical and microbial markers of periodontitis with Alzheimer disease incidence and mortality ${ }^{91}$. A plausible mechanism underlying the link between periodontitis and Alzheimer disease might be the infiltration of the brain with periodontal pathogens, a hypothesis supported by emerging evidence implicating $P$. gingivalis in particular. P. gingivalis DNA has been detected in Alzheimer disease brain autopsy specimens and the cerebrospinal fluid of living individuals with likely Alzheimer disease diagnosis ${ }^{11}$. Also detectable in Alzheimer disease brain autopsies are the pathogen's gingipains, the abundance of which correlates with tau protein load and ubiquitin load, markers of Alzheimer disease pathology ${ }^{11}$. In vitro, the gingipains can cleave tau ${ }^{11}$, an activity that might contribute to aberrant phosphorylation of tau and accumulation of insoluble tau forms in Alzheimer disease $^{92}$ (FIG. 5b).

Consistent with these clinical and laboratory observations, the brains of aged (11-month-old) mice, which were orally infected with $P$. gingivalis, showed DNAbased evidence of pathogen infiltration and increased levels of amyloid- $\beta_{1-42}$, a component of amyloid plaques ${ }^{11}$ (FIG. 5b). By contrast, amyloid- $\beta_{1-42}$ was not elevated (relative to the baseline of mock infection) when mice were infected with gingipain-deficient $P$. gingivalis or with wild-type $P$. gingivalis together with an orally administered gingipain inhibitor ${ }^{11}$. Gingipain inhibition also reduced the $P$. gingivalis load and neuroinflammation and prevented loss of hippocampal neurons ${ }^{11}$. It remains uncertain how $P$. gingivalis gains access to the brain, although its ability to cause increased vascular endothelial permeability ${ }^{15}$ (FIG. 5a) may facilitate its passage through the blood-brain barrier.

The apolipoprotein E gene ( $A P O E$ ) (particularly the $\mathrm{E} 4$ allele) is the strongest genetic risk factor for late-onset Alzheimer disease. APOE is implicated in the metabolism, aggregation and deposition of amyloid- $\beta^{93}$. APOE fragmentation may have neurotoxic effects ${ }^{94}$, and $P$. gingivalis gingipains cleave APOE at Arg residues ${ }^{95}$, suggesting an additional possible mechanism whereby $P$. gingivalis might contribute to Alzheimer disease. P. gingivalis can cause neuroinflammation also in the absence of APOE. Oral infection of $A p o E^{-/-}$mice with $P$. gingivalis, but not with two other important periodontal pathogens (Treponema denticola and Tannerella forsythia), resulted in brain infection, complement activation and neuronal injury $^{96}$ (FIG. 5b). Aberrant complement activation, which is associated with Alzheimer disease pathogenesis ${ }^{97}$, may be facilitated in the absence of APOE (or in the presence 

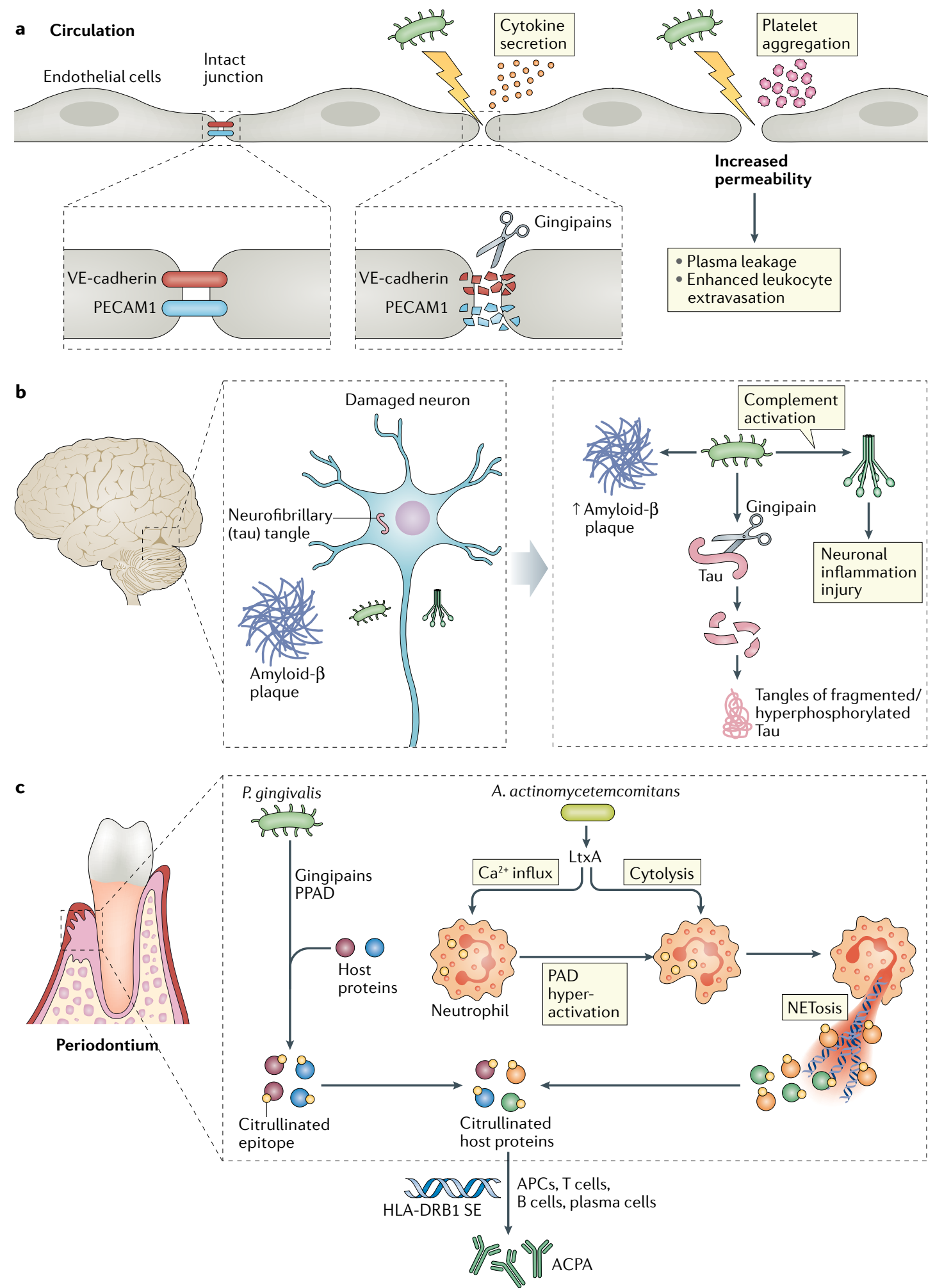

of defective APOE). Indeed, human APOE binds with high affinity to activated $\mathrm{Clq}$, the initiator of the classical complement cascade, and the resulting C1q-APOE complexes (which are detected in amyloid- $\beta$ plaques) downregulate complement activity ${ }^{98}$.

The risk of Alzheimer disease-related mortality in individuals 65 years or older is increased when the mortality association with anti-periodontal pathogen IgG titres at baseline involves clusters of bacteria rather than individual bacteria ${ }^{91}$. In addition to $P$. gingivalis, such clusters include Campylobacter rectus and Prevotella melaninogenica among other bacteria ${ }^{91}$. Thus, an interesting question is whether and, if so, how P. gingivalis cooperates with other bacteria to possibly contribute to Alzheimer disease pathology, as it does in periodontitis ${ }^{87}$. 
4 Fig. 5 | Mechanisms of periodontal bacterial action in extra-oral pathologies. a | Porphyromonas gingivalis causes vascular endothelial barrier disruption and increased permeability by disrupting intercellular junctions. This action is mediated by its gingipain proteases, which degrade platelet endothelial cell adhesion molecule (PECAM1) and vascular endothelial cadherin (VE-cadherin), which are crucial for junctional integrity. Endothelial damage and elevated permeability may instigate several processes that potentially induce or exacerbate atherogenesis, including induction of pro-inflammatory cytokines, platelet aggregation and increased leukocyte extravasation to subendothelial areas. $\mathbf{b} \mid$ P. gingivalis-induced pathology in Alzheimer disease. P. gingivalis DNA and gingipains have been detected in brain autopsies of patients with Alzheimer disease as well as in the brain of mice orally infected with this pathogen. The presence of $P$. gingivalis in the mouse brain is associated with increased levels of amyloid- $\beta$, complement activation and neuroinflammation. The gingipains of the pathogen cleave the microtubuleassociated protein tau, an activity that promotes aberrant phosphorylation of tau and accumulation of misfolded insoluble tau in Alzheimer disease. $\mathbf{c}$ | Role of periodontal bacteria in the generation of anti-citrullinated protein antibodies (ACPAs). P. gingivalis expresses a unique (among prokaryotic organisms) peptidyl-arginine deiminase (PPAD), which can citrullinate proteins including host proteins. The PPAD activity is facilitated by the pathogen's arginine-specific gingipains, which cleave proteins and expose C-terminal arginine residues for citrullination by PPAD. Alternatively, Aggregatibacter actinomycetemcomitans indirectly causes host protein citrullination by secreting leukotoxin A (LtxA), a pore-forming toxin that induces calcium influx and hyperactivation of PAD enzymes in neutrophils, as well as cytolysis (by NETosis, a form of cell death typified by the release of decondensed chromatin and granular contents to the extracellular milieu), thereby releasing the generated citrullinated autoantigens. Thus, through distinct mechanisms, both pathogens can contribute to the generation of the rheumatoid arthritis-specific ACPAs that promote disease in individuals with HLA-DRB1 shared epitope (SE) alleles. APC, antigen-presenting cell.

\footnotetext{
Pathobionts

Organisms that are generally benign or commensal but that have the capacity to promote pathology under specific conditions of disrupted host-microbiota homeostasis (for example, resulting from immune deficiencies, antibiotic treatment, tissue damage or dietary shifts).
}

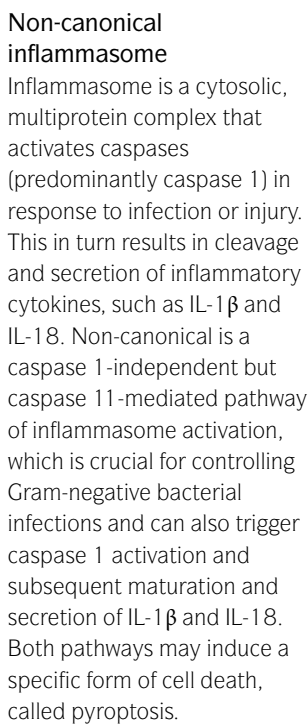

It should be noted that $P$. gingivalis may not be an obligatory mechanism for the connection between periodontitis and Alzheimer disease. In a mouse model of Alzheimer disease, induction of experimental periodontitis in the absence of $P$. gingivalis (by using the LIP model; BOX 1 ) increased the levels of insoluble amyloid- $\beta$ and affected neuroinflammation, both of which were attenuated by anti-inflammatory treatment ${ }^{99,100}$. Although direct involvement of indigenous mouse oral bacteria cannot be formally ruled out (in a manner similar to that of $P$. gingivalis), these findings raise the non-mutually exclusive possibility that periodontitis could contribute to Alzheimer disease pathology also by increasing systemic inflammation.

Inflammatory bowel disease. IBD is associated with increased risk of periodontitis ${ }^{101}$. Consistently, SAMP1/ YitFc mice, a spontaneous model of Crohn's disease, develop natural periodontitis ${ }^{102}$. Recent animal studies suggest that periodontal pathogens can contribute to intestinal inflammation by influencing gut dysbiosis and barrier function ${ }^{6,8,9,36}$, implying a bidirectional association between the two disorders.

Mouse periodontitis induced by oral gavage with P. gingivalis alone or with other periodontal pathogens (F. nucleatum and $P$. intermedia) alters the gut microbiota ${ }^{9,36}$ and results in reduced expression of epithelial tight-junction proteins in the ileum and increased expression of proinflammatory cytokines in the colon ${ }^{36}$. As $P$. gingivalis was only transiently detected in the gut, where it failed to colonize $e^{36}$, the precise underlying mechanisms in this pioneering study are largely uncertain.

Advanced mechanistic understanding was achieved in a recent study that used the LIP model, which leads to expansion of indigenous oral pathobionts ${ }^{6}$. A subset of oral pathobionts (predominantly comprising Klebsiella spp. and Enterobacter spp.) was shown to ectopically colonize the mouse gut, where they promoted colitis through induction of non-canonical inflammasome-dependent IL- $1 \beta$ secretion by inflammatory macrophages (Ly6C ${ }^{\text {lo }} \mathrm{MHC}-\mathrm{II}^{\text {hi }} \mathrm{CD} 64^{\text {hi }}$ ) (FIG. 4). Gut colonization by oral pathobionts and promotion of colitis required pre-existing intestinal inflammation (induced by dextran sodium sulfate treatment) or a colitis-susceptible host, specifically IL-10-deficient mice ${ }^{6}$, which mimic IBD-susceptible humans with specific IL-10R polymorphisms ${ }^{103}$.

Human oral pathobionts can also promote colitis in animal models. Germ-free mice gavaged with oral (salivary) microbiota from a patient with Crohn's disease displayed expansion of intestinal T helper $1\left(\mathrm{~T}_{\mathrm{H}} 1\right)$ IFN $\gamma^{+} \mathrm{CD}^{+}$cells, which was attributed to Klebsiella spp. (Klebsiella pneumoniae and Klebsiella aeromobilis) ${ }^{8}$. Ectopic gut colonization by oral K. pneumoniae $2 \mathrm{H} 7$ invariably resulted in expansion of $\mathrm{T}_{\mathrm{H}} 1$ cells, although induction of gut inflammation additionally required a colitis-susceptible host, specifically germ-free IL-10-deficient mice ${ }^{8}$. The K. pneumoniae $2 \mathrm{H} 7$-induced $\mathrm{T}_{\mathrm{H}} 1$ cell response required the presence of the intestinal CD $11 \mathrm{~b}^{-} \mathrm{CD} 103^{+} \mathrm{DC}$ subset, possibly acting as antigen-presenting cells, and TLR4-activated colonic epithelial cells secreting IL-18 appeared to amplify the $\mathrm{T}_{\mathrm{H}} 1$ cell response ${ }^{8}$.

As discussed above, LIP in wild-type mice does not lead to gut colonization by oral pathobionts in the absence of pre-existing colitis pathology ${ }^{6}$. Moreover, wild-type mice are resistant to colonization by K. pneumoniae $2 \mathrm{H} 7$ unless they are treated with antibiotics ${ }^{8}$. These findings suggest that a normal gut flora can prevent ectopic colonization by oral bacteria, unless it is disrupted by local inflammation or by antibiotics. Similarly, although humans daily swallow 1.51 of saliva containing approximately $\sim 10^{12}$ oral bacteria ${ }^{36,104}$, oral microorganisms are generally poor colonizers of the healthy human intestine; by contrast, oral microorganisms are enriched in the gut microbiota of patients with IBD, colon cancer, liver cirrhosis and other diseases ${ }^{35,85,86,104,105}$. In fact, most of the dominant species with increased abundance in paediatric Crohn's disease or ulcerative colitis are normally inhabitants of the oral cavity (such as Fusobacteriaceae and Veillonellaceae) rather than typical intestinal organisms ${ }^{85,86}$. Importantly, increased abundance of oral bacteria correlates with disease severity, suggesting a potential role of oral species in IBD pathogenesis or progression ${ }^{85,86}$.

Colorectal cancer. The gut microbiota of patients with colorectal cancer (CRC) exhibits increased species richness and abundance (compared with healthy controls), and this is attributed in part to ectopic colonization and expansion of bacteria deriving from the oral cavity (for instance, F. nucleatum, Parvimonas micra, Peptostreptococcus stomatis, Solobacterium moorei and Porphyromonas asaccharolytica $)^{105-108}$. The evidence for involvement in human CRC is particularly strong for F. nucleatum ${ }^{109,110}$, an oral bacterium that has also been implicated in pregnancy complications in both humans and experimental mouse models, where it can reach the 
fetal-placental compartment by the haematogenous route $^{111}$ (FIG. 1). Specifically, F. nucleatum can promote the growth ${ }^{112}$ and migration ${ }^{113}$ of human CRC cells and their resistance to chemotherapy ${ }^{114}$, as well as enhance intestinal tumorigenesis in the $A p c^{\mathrm{Min} /+}$ mouse model ${ }^{107}$.

The notion that CRC-associated F. nucleatum originates from the oral cavity is supported by findings that identical strains of this organism are detected in the saliva and colorectal tumours of patients with $\mathrm{CRC}^{115}$. Oral F. nucleatum can haematogenously reach the colon ${ }^{116}$, where it exhibits tropism for CRC. This is because CRC cells overexpress a carbohydrate moiety (Gal-GalNAc) that is bound by the F. nucleatum lectin Fap2 (REF.7), which also induces the release of prometastatic chemokines (IL- 8 and CXC-chemokine ligand 1 (CXCL1)) that drive CRC migration ${ }^{113}$ and tumour-related angiogenesis ${ }^{117}$. Another $F$. nucleatum adhesin, FadA, stimulates the growth of CRC (but not of non-cancerous) cells by inducing E-cadherin-mediated Wnt- $\beta$-catenin signalling in a manner dependent on annexin $\mathrm{A} 1$, which is upregulated in $\mathrm{CRC}^{112,118}$. Moreover, F. nucleatum alters the tumour microenvironment in a manner that facilitates CRC progression; specifically, it recruits tumour-infiltrating myeloid-derived suppressor cells ${ }^{107}$ and undermines the cytotoxic function of tumour-infiltrating T cells and natural killer cells ${ }^{10}$. In the latter mechanism, F. nucleatum uses its Fap2 adhesin to activate the inhibitory immunoreceptor T cell immunoreceptor with immunoglobulin and ITIM domains (TIGIT), thereby enabling the tumour cells to evade killing by $\mathrm{T}$ cells and natural killer cells ${ }^{10}$. Besides $F$. nucleatum, several other oral bacteria are suspected to play a role in different types of carcinoma (reviewed in REF. ${ }^{119}$ ).

Locally acting periodontal bacteria with remote consequences. Periodontitis is associated with increased disease activity in patients with rheumatoid arthritis ${ }^{120,121}$, and local periodontal treatment reduces the severity of arthritis ${ }^{122-124}$. Although periodontitis-associated systemic inflammation likely contributes to this association, two periodontal pathogens may independently contribute to the periodontitis-rheumatoid arthritis connection. Specifically, through distinct mechanisms, $P$. gingivalis and A. actinomycetemcomitans may promote the generation of the anti-citrullinated protein antibodies (ACPAs) that are seen in patients with rheumatoid arthritis ${ }^{2,12,125,126}$ (FIG. 5c). The disruption of immune tolerance to citrullinated proteins in rheumatoid arthritis-susceptible individuals is associated with HLA-DRB1 alleles encoding a shared epitope that binds selectively to citrullinated peptides. Citrullinated proteins are found in higher abundance in the inflamed periodontium than in healthy tissue, and ACPAs are detectable in the circulation prior to rheumatoid arthritis symptom onset and correlate with disease severity ${ }^{2,12}$.

$P$. gingivalis is uniquely equipped, among tested prokaryotes, with a peptidyl-arginine deiminase (PAD) that citrullinates proteins including human fibrinogen and $\alpha$-enolase, which are major rheumatoid arthritis autoantigens in their citrullinated form ${ }^{2,127}$. The P. gingivalis citrullination capacity is facilitated by its arginine-specific gingipains, which cleave fibrinogen and $\alpha$-enolase exposing $\mathrm{C}$-terminal arginine residues that can be citrullinated by $P$. gingivalis $\mathrm{PAD}(\mathrm{PPAD})^{127}$. In patients with ACPA-positive rheumatoid arthritis, the detection of $P$. gingivalis in the periodontal biofilm correlates with increased levels of ACPAs ${ }^{121}$. Infection of mice with wild-type, but not PPAD-deficient, strains of $P$. gingivalis aggravates collagen- or collagen antibody-induced arthritis, accompanied by the presence of citrullinated proteins at the infection site and generation of ACPAs ${ }^{125,126}$. Antibiotic treatment of mice subjected to $P$. gingivalis-induced periodontitis inhibited subsequent induction of collagen-induced arthritis in the same animals ${ }^{128}$. However, it has also been proposed that orally administered $P$. gingivalis can aggravate experimental rheumatoid arthritis in mice independently of its citrullinating activity, presumably by altering the gut microbiota, impairing gut barrier function and elevating systemic inflammation ${ }^{14,129}$.

Although A. actinomycetemcomitans does not encode a PAD-like enzyme, it induces hypercitrullination of host proteins indirectly by secreting a pore-forming toxin, leukotoxin A (LtxA), that binds to $\beta 2$ integrin adhesion receptors on neutrophils inducing calcium influx and aberrant hyperactivation of the neutrophil's PAD enzymes $^{12}$. Moreover, LtxA causes the release of citrullinated autoantigens through cytolysis ${ }^{12}$ (FIG. 5c). Because the toxicity of LtxA is restricted to leukocytes from primates, it has not been possible to test whether A. actinomycetemcomitans can induce an ACPA response in vivo using currently available rodent models. However, the human relevance of the proposed mechanism is supported by findings that the concentration of anti-LtxA antibodies is strongly associated with ACPA positivity, especially when the patients are analysed in the context of HLA-DRB1 shared epitope alleles ${ }^{12}$.

\section{Trafficking of orally primed inflammatory cells}

Lymphocyte trafficking. The concept that immune responses primed at a certain mucosal barrier site can be effectively recalled at other mucosal sites is a hallmark of the common mucosal immune system and forms the basis of mucosal vaccination-induced protection against pathogens at both local and remote sites ${ }^{130,131}$. However, the same mechanism may have detrimental effects and link two distinct mucosal inflammatory diseases.

Indeed, $\mathrm{T}$ cell priming in the periodontal tissue and subsequent $\mathrm{T}$ cell trafficking to the gut links periodontitis to colitis in mice ${ }^{6}$ (FIG. 4). Specifically, oral pathobiont-specific T cells, which expand during LIP, express the gut-homing molecules CC-chemokine receptor 9 (CCR9) and $\alpha 4 \beta 7$ integrin (the receptor for the gut-specific vascular addressin MAdCAM1) and migrate from the oral cavity-draining cervical lymph nodes to the gut ${ }^{6}$. Once in the gut, these oral pathobiont-specific $\mathrm{T}$ cells, in which $\mathrm{T}_{\mathrm{H}} 17$ cells are enriched, proliferate and exacerbate colitis ${ }^{6}$ (FIG. 4). Interestingly, a monoclonal antibody to the $\alpha 4 \beta 7$ integrin (vedolizumab), which has been approved for the treatment of IBD $^{132}$, might in part protect against IBD by inhibiting the trafficking of orally primed $\mathrm{T}$ cells to the gut.

Once in the gut, these $\mathrm{T}$ cells of oral origin proliferate in response to their cognate antigens, because - as 
discussed above - oral pathobionts can ectopically colonize the gut ${ }^{6}$. Moreover, the ectopic oral pathobionts stimulate intestinal macrophages to secrete IL- $1 \beta$, which contributes to the expansion of the transmigrated oral $\mathrm{T}_{\text {cells }}{ }^{6}$ (FIG. 4). In the gut, these colitogenic T cells of oral origin display ROR $\gamma \mathrm{t}^{+}$and ROR $\gamma \mathrm{t}^{+} \mathrm{T}$-bet ${ }^{+} \mathrm{T}_{\mathrm{H}} 17$ cell phenotypes and, therefore, secrete both IL-17A and IFN $\gamma^{6}$. This experimental finding is consistent with clinical observations showing the presence of IL-17A and IFN $\gamma$ and both $\mathrm{T}_{\mathrm{H}} 17$ cells and cells with a mixed $\mathrm{T}_{\mathrm{H}} 17 / \mathrm{T}_{\mathrm{H}} 1$ phenotype in the intestinal mucosa of patients with $\mathrm{IBD}^{133}$.

Consistent with the study discussed above ${ }^{6}$ and the concept that the mucosal immune system is an integrated, system-wide organ ${ }^{131}$, an earlier study showed that respiratory influenza infection in mice results in intestinal immune injury attributed to the recruitment of CCR9 ${ }^{+} \mathrm{CD}^{+} \mathrm{T}$ cells from the lungs ${ }^{134}$. The lung-derived transmigrated $\mathrm{T}$ cells caused intestinal dysbiosis and inflammation by secreting IL-17A and IFN $\gamma^{134}$. Although not all individuals with flu, or other respiratory infections, develop diarrhoea or other gastroenteritis symptoms, this mechanism might contribute to those substantial cases presenting with both respiratory and gastroenteritis symptoms, as seen in many patients with COVID-19 (REF. ${ }^{135}$ ).

Myeloid cell trafficking. As alluded to above, blood-borne $P$. gingivalis is taken up by human blood $\mathrm{CD} 1 \mathrm{c}^{+} \mathrm{CD} 209^{+}$ $\mathrm{mDC}$ through an interaction between its Mfal fimbrial protein and the C-type lectin DC-specific ICAM3grabbing non-integrin (DC-SIGN) ${ }^{84}$. Interestingly, $P$. gingivalis appears to modulate the trafficking and behaviour of mDCs in a manner consistent with the detection of $P$. gingivalis-carrying $\mathrm{mDCs}$ in atheromatous plaques $^{84}$. In response to internalized $P$. gingivalis, human monocyte-derived DCs selectively upregulate the expression of CXC-chemokine receptor 4 (CXCR4), which directs homing to atheromatous plaques, whereas they do not upregulate the CCR7, which mediates homing to secondary lymphoid organs ${ }^{136,137}$. Consistently, peripheral blood mDCs from patients with periodontitis display higher CXCR4 and lower CCR7 expression relative to their counterparts from healthy controls ${ }^{136}$. Moreover, human monocyte-derived DCs that have internalized $P$. gingivalis acquire enhanced pro-inflammatory and atherogenic potential (for example, they secrete high levels of MMP9, an indicator of plaque rupture risk $)^{84}$.

The P. gingivalis-carrier $\mathrm{CD} 1 \mathrm{c}^{+} \mathrm{CD} 209^{+} \mathrm{mDCs}$ moreover downregulate apoptosis ${ }^{13}$, expand in the blood of patients with periodontitis ${ }^{84}$ and promote systemic $\mathrm{T}_{\mathrm{H}} 17$ cell responses ${ }^{138}$, thereby potentially influencing not only CVD but also IL-17-driven systemic conditions. The inhibition of DC apoptosis (which is generally linked to aberrant inflammatory conditions ${ }^{139}$ ) by $P$. gingivalis depends on MFal fimbria-induced activation of a DC-SIGN-AKT-mTOR anti-apoptotic pathway ${ }^{13}$.

The microbial cargo of $P$. gingivalis-carrier blood mDCs contains additional species (Burkholderia cepacia, Helicobacter pylori, Pseudomonas spp., Moraxella catarrhalis, K. pneumoniae and Salmonella enterica) ${ }^{84}$. Therefore, additional species, not necessarily of oral origin, might contribute to the pathological reprogramming of these cells. However, as the expansion of these carrier mDCs in human blood increases significantly after debridement-induced bacteraemia ${ }^{84}$, periodontitis-associated pathogens are likely important for their altered pathogenic potential.

\section{Conclusions and outlook}

Epidemiological, clinical interventional and experimental studies collectively offer sufficient evidence that periodontitis adversely impacts systemic health through biologically plausible mechanisms (FICS 2-5). Although clinical intervention studies suggest that local periodontal treatment decreases the serum levels of inflammatory factors and improves metabolic control, lipid profile and other surrogate markers of systemic disease ${ }^{5,19,22,29,30,32,33,42,43,122,123}$, clear evidence that successful treatment of periodontal disease can reduce the risk or incidence of epidemiologically associated conditions is lacking. Such evidence would require multicentre randomized controlled trials and could establish that periodontitis is a modifiable risk factor for life-threatening comorbidities. In this regard, further improvement of local periodontal therapy through adjunctive hostmodulation treatments (such as by targeting complement or pro-resolution pathways ${ }^{140}$ ) may contribute to reducing systemic inflammation and promote systemic health. Moreover, targeted therapies against specific periodontal pathogens (such as $P$. gingivalis and $F$. nucleatum) or the key virulence factors they produce (for example, gingipains and Fap2) might ameliorate specific comorbidities, such as Alzheimer disease and CRC.

Future translational research should importantly target the mechanistic understanding of the connection between periodontitis and comorbidities. The notion that inflammation-adapted haematopoietic progenitors may link periodontitis and CVD is consistent with clinical-imaging correlation of periodontal inflammation with haematopoietic tissue activity and arterial inflammation ${ }^{20}$ but will require experimental confirmation in a broader context involving additional comorbidities (for example, rheumatoid arthritis, T2DM and NAFLD). Blocking key mediators of this process may reduce the risk of multiple comorbidities. Given that IL- $1 \beta$ is crucial for the induction of innate immune memory, the generation of inflammation-adapted HSPCs and enhanced myelopoiesis in cardiometabolic disease ${ }^{18}$, systemic inhibition of this cytokine may protect not only against atherosclerotic CVD (as shown by the CANTOS trial ${ }^{141}$ ) but also against several inflammatory comorbidities. Inhibition of IL- $1 \beta$ may potentially disrupt a common mechanistic basis for inflammatory comorbidities, namely the maladaptive training of bone marrow progenitors. Intriguingly, IL- $1 \beta$ and IL- 6 also seem to link the age-related clonal haematopoiesis of indeterminate potential (CHIP) to exacerbation of inflammation in $\mathrm{CVD}^{142,143}$ and perhaps other ageing-related inflammatory disorders, such as periodontitis ${ }^{18}$. CHIP is likely both a driver and a consequence of inflammation ${ }^{18,142,143}$ and, as such, may be associated with periodontitis, although this has yet to be addressed by clinical and experimental studies. 
At least in principle, reversing the pathological effects of CHIP may ameliorate the severity of periodontitis and associated systemic comorbidities.

The emerging findings that inflammatory processes in various peripheral tissues ('peripheral' inflammation) may be interlinked via inflammatory adaptations of the bone marrow as a central hub ('central' inflammation) ) $^{18}$ may not only improve our mechanistic understanding of comorbidities but also revolutionize the way scientists view inflammation and disease. Specifically, factors regulating peripheral inflammation may in fact do so by additionally modulating central inflammatory processes. For instance, the homeostatic protein developmental endothelial locus 1 (DEL1; also known as EGF-like repeat and discoidin I-like domain-containing protein 3 ), which was shown to inhibit initiation of periodontal inflammation and to promote its resolution ${ }^{144-147}$, may also regulate central inflammatory processes via its function to modulate demand-adapted myelopoiesis in the bone marrow ${ }^{148}$.

Furthermore, in the light of these emerging mechanisms as bases of comorbidities (for example, trained immunity and CHIP), future clinical studies investigating the effects of improving periodontal health on systemic comorbidities should identify and use surrogate markers of those mechanisms (for instance, myelopoiesis output in the peripheral blood) and novel risk-assessment scores. We clearly need unifying frameworks, in which peripheral inflammatory and infectious stimuli (such as those associated with periodontitis) and multiple systemic risk factors (such as, obesity, dyslipidaemia, glycaemic level and ageing) can be productively integrated to better understand the interconnected pathogenesis of distinct chronic inflammatory diseases and/or malignant disorders. Inflammatory adaptation of haematopoietic progenitors represents such a unifying framework between peripheral and central inflammation that could also provide the platform for novel therapeutic interventions targeting inflammation and inflammatory comorbidities via a holistic approach, although this concept has to be addressed in future investigations.

Published online 28 January 2021
1. Genco, R. J. \& Sanz, M. Clinical and public health implications of periodontal and systemic diseases: an overview. Periodontol. 2000 83, 7-13 (2020).

2. Potempa, J., Mydel, P. \& Koziel, J. The case for periodontitis in the pathogenesis of rheumatoid arthritis. Nat. Rev. Rheumatol. 13, 606-620 (2017).

3. Hajishengallis, G. Periodontitis: from microbial immune subversion to systemic inflammation. Nat. Rev. Immunol. 15, 30-44 (2015).

4. Acharya, C., Sahingur, S. E. \& Bajaj, J. S. Microbiota, cirrhosis, and the emerging oral-gut-liver axis. JCI Insight 2, e94416 (2017).

5. Schenkein, H. A., Papapanou, P. N., Genco, R. \& Sanz, M. Mechanisms underlying the association between periodontitis and atherosclerotic disease Periodontol. 2000 83, 90-106 (2020).

6. Kitamoto, $\mathrm{S}$. et al. The intermucosal connection between the mouth and gut in commensal pathobiontdriven colitis. Cell 182, 447-462 (2020). This study elegantly shows that oral bacterialspecific $T_{H} 17$ cells, which expand during experimental periodontitis, are instructed to migrate to the gut, where they are activated by translocated (via the gastrointestinal route) oral bacteria and contribute to the development of colitis.

7. Abed, J. et al. Fap2 mediates Fusobacterium nucleatum colorectal adenocarcinoma enrichment by binding to tumor-expressed Gal-GalNAc. Cell Host Microbe 20, 215-225 (2016). This paper explains $F$. nucleatum's tropism to CRC. The bacterium expresses a Gal-GalNAcbinding lectin (Fap2) that can bind to the host polysaccharide Gal-GalNAc, which is highly expressed in human CRC

8. Atarashi, K. et al. Ectopic colonization of oral bacteria in the intestine drives TH 1 cell induction and inflammation. Science 358, 359-365 (2017). This is the first paper to show that ectopic gut colonization by bacteria of oral origin results in expansion of colitogenic $T$ cells and promotion of colitis in a susceptible host.

9. Blasco-Baque, V. et al. Periodontitis induced by Porphyromonas gingivalis drives periodontal microbiota dysbiosis and insulin resistance via an impaired adaptive immune response. Gut 66, 872-885 (2017)

10. Gur, C. et al. Binding of the Fap2 protein of Fusobacterium nucleatum to human inhibitory receptor TIGIT protects tumors from immune cell attack. Immunity 42, 344-355 (2015). This study identifies an important immune evasion mechanism by $F$. nucleatum, which uses its Fap 2 adhesin to activate the inhibitory immunoreceptor TIGIT, which disables the tumour-killing activity of T cells and natural killer cells.

11. Dominy, S. S. et al. Porphyromonas gingivalis in Alzheimer's disease brains: evidence for disease causation and treatment with small-molecule inhibitors. Sci. Adv. 5, eaau3333 (2019).

This study provides strong clinical and experimental evidence that the oral pathogen $P$. gingivalis can infect the brain and contribute to inflammatory pathology in Alzheimer disease.

12. Konig, M. F. et al. Aggregatibacter

actinomycetemcomitans-induced hypercitrullination links periodontal infection to autoimmunity in rheumatoid arthritis. Sci. Transl. Med. 8, 369ra 176 (2016).

In this study, the periodontal pathogen

A. actinomycetemcomitans is shown to induce the generation of citrullinated autoantigens involved in rheumatoid arthritis through the action of its pore-forming leukotoxin A (LtXA) on neutrophils. Anti-LtxA antibodies are strongly associated with ACPA positivity in patients with rheumatoid arthritis.

13. Meghil, M. M. et al. Disruption of immune homeostasis in human dendritic cells via regulation of autophagy and apoptosis by Porphyromonas gingivalis. Front. Immunol. 10, 2286 (2019).

14. Sato, K. et al. Aggravation of collagen-induced arthritis by orally administered Porphyromonas gingivalis through modulation of the gut microbiota and gut immune system. Sci. Rep. 7, 6955 (2017).

15. Farrugia, C. et al. Mechanisms of vascular damage by systemic dissemination of the oral pathogen Porphyromonas gingivalis. FEBS J. https://doi.org/ 10.1111/febs. 15486 (2020)

16. Zhao, Y. et al. Characterization and regulation of osteoclast precursors following chronic Porphyromonas gingivalis infection. J. Leukoc. Biol. 108, 1037-1050 (2020).

This investigation identifies a mechanism whereby the bone marrow may link periodontitis to other bone loss disorders. Specifically, periodontal bacteria-induced serum IL- 6 acts in the bone marrow to promote the expansion and osteoclastogenic lineage bias of a precursor population, which can traffic to sites of bone resorption and differentiate into mature osteoclasts.

17. Netea, M. G. et al. Defining trained immunity and its role in health and disease. Nat. Rev. Immunol. 20, 375-388 (2020)

18. Chavakis, T., Mitroulis, I. \& Hajishengallis, G. Hematopoietic progenitor cells as integrative hubs for adaptation to and fine-tuning of inflammation. Nat. Immunol. 20, 802-811 (2019).

19. D'Aiuto, F. et al. Systemic effects of periodontitis treatment in patients with type 2 diabetes: a 12 month, single-centre, investigator-masked, randomised trial. Lancet Diabetes Endocrinol. 6 954-965 (2018) A well-designed and executed randomized controlled trial showing favourable effects of local periodontal treatment on systemic inflammatory markers, glycaemic control and vascular and kidney functions in patients with type $\mathbf{2}$ diabetes mellitus.

20. Ishai, A. et al. Periodontal disease associates with arterial inflammation via potentiation of a hematopoietic-arterial axis. JACC Cardiovasc. Imaging 12, 2271-2273 (2019).

An important clinical-imaging study that correlates periodontal metabolic activity/inflammation with haematopoietic tissue activity and arterial inflammation, thus supporting the hypothesis derived from experimental studies that the inflammatory adaptation of haematopoietic progenitor cells in the bone marrow may link different chronic inflammatory diseases

21. Teles, R. \& Wang, C.-Y. Mechanisms involved in the association between peridontal diseases and cardiovascular disease. Oral Dis. 17, 450-461 (2011).

22. Bajaj, J. S. et al. Periodontal therapy favorably modulates the oral-gut-hepatic axis in cirrhosis. Am. J. Physiol. Gastrointest. Liver Physiol. 315, G824-G837 (2018).

23. Xiao, E. et al. Diabetes enhances IL-17 expression and alters the oral microbiome to increase its pathogenicity. Cell Host Microbe 22, 120-128 e124 (2017).

24. Teles, F., Wang, Y., Hajishengallis, G., Hasturk, H. \& Marchesan, J. Impact of systemic factors in shaping the periodontal microbiome. Periodontol. 200085 126-160 (2021).

25. Monsarrat, P. et al. Clinical research activity in periodontal medicine: a systematic mapping of trial registers. J. Clin. Periodontol. 43, 390-400 (2016).

26. Rydén, L. et al. Periodontitis increases the risk of a first myocardial infarction. Circulation 133, 576-583 (2016).

27. Rodríguez-Lozano, B. et al. Association between severity of periodontitis and clinical activity in rheumatoid arthritis patients: a case-control study. Arthritis Res. Ther. 21, 27 (2019).

28. Genco, R. J. \& Van Dyke, T. E. Prevention: reducing the risk of CVD in patients with periodontitis. Nat. Rev. Cardiol. 7, 479-480 (2010).

29. D’Aiuto, F., Orlandi, M. \& Gunsolley, J. C. Evidence that periodontal treatment improves biomarkers and CVD outcomes. J. Clin. Periodontol. 40, S85-S105 (2013).

30. H. Bokhari, S. A et al. Non-surgical periodontal therapy reduces coronary heart disease risk markers: a randomized controlled trial. J. Clin. Periodontol. 39, 1065-1074 (2012).

31. de Oliveira, C., Watt, R. \& Hamer, M. Toothbrushing, inflammation, and risk of cardiovascular disease: results from Scottish health survey. Br. Med. J. 340 c2451 (2010)

32. Tonetti, M. S. Periodontitis and risk for atherosclerosis: an update on intervention trials. J. Clin. Periodontol. 36, 15-19 (2009). 
33. Türer C, C., Durmus, D., Balli, U. \& Güven, B. Effect of non-surgical periodontal treatment on gingival crevicular fluid and serum endocan, vascular endothelial growth factor-A, and tumor necrosis factor-alpha levels. J. Periodontol. 88, 493-50 (2017).

34. Mammen, M. J., Scannapieco, F. A. \& Sethi, S. Oral-lung microbiome interactions in lung diseases. Periodontol. 2000 83, 234-241 (2020).

35. Qin, N. et al. Alterations of the human gut microbiome in liver cirrhosis. Nature 513, 59-64 (2014).

36. Arimatsu, K. et al. Oral pathobiont induces systemic inflammation and metabolic changes associated with alteration of gut microbiota. Sci. Rep. 4, 4828 (2014).

37. Hotamisligil, G. S. Inflammation and metabolic disorders. Nature 444, 860-867 (2006).

38. Norata, G. D. et al. The cellular and molecular basis of translational immunometabolism. Immunity 43, 421-434 (2015)

39. Orlandi, M., Graziani, F. \& D'Aiuto, F. Periodontal therapy and cardiovascular risk. Periodontol. 2000 83, 107-124 (2020)

40. Genco, R. J., Graziani, F. \& Hasturk, H. Effects of periodontal disease on glycemic control, complications, and incidence of diabetes mellitus. Periodontol. 2000 83, 59-65 (2020).

41. Jepsen, S., Suvan, J. \& Deschner, J. The association of periodontal diseases with metabolic syndrome and obesity. Periodontol. 2000 83, 125-153 (2020).

42. Tonetti, M. S. et al. Treatment of periodontitis and endothelial function. N. Engl. J. Med. 356, 911-920 (2007).

43. Desvarieux, M. et al. Changes in clinical and microbiological periodontal profiles relate to progression of carotid intima-media thickness: the oral infections and vascular disease epidemiology study. J. Am. Heart Assoc. 2, e000254 (2013).

44. Yoneda, M. et al. Involvement of a periodontal pathogen, Porphyromonas gingivalis on the pathogenesis of non-alcoholic fatty liver disease. BMC Gastroenterol. 12, 16 (2012).

45. Helenius-Hietala, J. et al. Periodontitis is associated with incident chronic liver disease - a populationbased cohort study. Liver Int. 39, 583-591 (2019).

46. Brito, L. C. W. et al. Experimental periodontitis promotes transient vascular inflammation and endothelial dysfunction. Arch. Oral. Biol. 58 1187-1198 (2013)

47. Matsuda, Y. et al. Ligature-induced periodontitis in mice induces elevated levels of circulating interleukin- 6 but shows only weak effects on adipose and liver tissues. J. Periodont. Res. 51, 639-646 (2016).

48. O'Boyle, C. et al. Ligature-induced periodontitis induces systemic inflammation but does not alter acute outcome after stroke in mice. Int. J. Stroke 15 175-187 (2019)

49. Anbinder, A. L. et al. Periodontal disease exacerbates systemic ovariectomy-induced bone loss in mice. Bone 83, 241-247 (2016)

50. Miyajima, S.-i. et al. Periodontitis-activated monocytes/macrophages cause aortic inflammation. Sci. Rep. 4, 5171 (2014).

51. Hasturk, H. et al. Resolvin E1 (RvE1) attenuates atherosclerotic plaque formation in diet and inflammation-induced atherogenesis. Arterioscl. Thromb. Vasc. Biol. 35, 1123-1133 (2015).

52. Tian, J. et al. Porphyromonas gingivalis induces insulin resistance by increasing BCAA levels in mice. J. Dent. Res. 99, 839-846 (2020).

53. White, P. J. \& Newgard, C. B. Branched-chain amino acids in disease. Science 363, 582-583 (2019).

54. Chavakis, T. et al. The pattern recognition receptor (RAGE) is a counterreceptor for leukocyte integrins: a novel pathway for inflammatory cell recruitment J. Exp. Med. 198, 1507-1515 (2003).

55. Ruiz, H. H., Ramasamy, R. \& Schmidt, A. M. Advanced glycation end products: building on the concept of the "Common Soil" in metabolic disease. Endocrinology 161, 1-10 (2020).

56. Lalla, E. et al. Blockade of RAGE suppresses periodontitis-associated bone loss in diabetic mice. J. Clin. Invest. 105, 1117-1124 (2000).

57. Nakahara, T. et al. Involvement of Porphyromonas gingivalis in the progression of non-alcoholic fatty liver disease. J. Gastroenterol. 53, 269-280 (2018).

58. Nagasaki, A. et al. Odontogenic infection by Porphyromonas gingivalis exacerbates fibrosis in NASH via hepatic stellate cell activation. Sci. Rep. 10 4134 (2020)

59. Vasconcelos, D. F. P. et al. Decrease of pericytes is associated with liver disease caused by ligature-induced periodontitis in rats. J. Periodontol. 88, e49-e57 (2017).

60. Tsukasaki, M. et al. Host defense against ora microbiota by bone-damaging T cells. Nat. Commun 9, 701 (2018)

61. Komazaki, R. et al. Periodontal pathogenic bacteria, Aggregatibacter actinomycetemcomitans affect non-alcoholic fatty liver disease by altering gut microbiota and glucose metabolism. Sci. Rep. 7 , 13950 (2017).

62. King, K. Y. \& Goodell, M. A. Inflammatory modulation of HSCs: viewing the HSC as a foundation for the immune response. Nat. Rev. Immunol. 11, 685 (2011).

63. Yamamoto, R. et al. Clonal analysis unveils selfrenewing lineage-restricted progenitors generated directly from hematopoietic stem cells. Cell 154 1112-1126 (2013).

64. Tsukasaki, M. \& Takayanagi, H. Osteoimmunology: evolving concepts in bone-immune interactions in health and disease. Nat. Rev. Immunol. 19, 626-642 (2019).

65. Herrera, B. S. et al. Peripheral blood mononuclear phagocytes from patients with chronic periodontitis are primed for osteoclast formation. J. Periodontol. 85, e72-e81 (2014).

66. Manz, M. G. \& Boettcher, S. Emergency granulopoiesis. Nat. Rev. Immunol. 14, 302-314 (2014).

67. Arts, R. J. W. et al. BCG vaccination protects against experimental viral infection in humans through the induction of cytokines associated with trained immunity. Cell Host Microbe 23, 89-100 (2018).

68. Mitroulis, I. et al. Modulation of myelopoiesis progenitors is an integral component of trained immunity. Cell 172, 147-161 (2018).

This study shows that trained immunity can be initiated in the bone marrow through long-lasting metabolic and transcriptional adaptations in HSPCs that lead to enhanced myelopoiesis.

69. Pietras, E. M. et al. Chronic interleukin-1 exposure drives haematopoietic stem cells towards precocious myeloid differentiation at the expense of self-renewal. Nat. Cell Biol. 18, 607-618 (2016)

70. Fifer, K. M. et al. Positron emission tomography measurement of periodontal 18F-fluorodeoxyglucose uptake is associated with histologically determined carotid plaque inflammation. J. Am. Coll. Cardiol. 57, 971-976 (2011)

71. Ling, M. R., Chapple, I. L. \& Matthews, J. B. Peripheral blood neutrophil cytokine hyper-reactivity in chronic periodontitis. Innate Immun. 21, 714-725 (2015).

72. Radvar, M., Tavakkol-Afshari, J., Bajestan, M. N., Naseh, M. R. \& Arab, H. R. The effect of periodontal treatment on IL- 6 production of peripheral blood monocytes in aggressive periodontitis and chronic periodontitis patients. Iran. J. Immunol. 5, 100-106 (2008).

73. Kleinnijenhuis, J. et al. Bacille Calmette-Guerin induces NOD2-dependent nonspecific protection from reinfection via epigenetic reprogramming of monocytes. Proc. Natl Acad. Sci. USA 109 17537-17542 (2012)

74. Cirovic, B. et al. BCG vaccination in humans elicits trained immunity via the hematopoietic progenitor compartment. Cell Host Microbe 28, 322-334 (2020).

This study shows that BCG vaccination in humans induces sustained transcriptomic myeloid bias in HSPCs associated with long-term heightened responsiveness of circulating myeloid cells to inflammatory stimuli, thereby providing human relevance for the findings of Mitroulis et al. (2018).

75. Bernelot Moens, S. J. et al. Unexpected arterial wall and cellular inflammation in patients with rheumatoid arthritis in remission using biological therapy: a cross-sectional study. Arthritis Res. Ther. 18, 115 (2016).

76. Schloss, M. J., Swirski, F. K. \& Nahrendorf, M. Modifiable cardiovascular risk, hematopoiesis, and innate immunity. Circ. Res. 126, 1242-1259 (2020).

77. Christ, A. et al. Western diet triggers NLRP3dependent innate immune reprogramming. Cell 172 162-175 (2018)

78. Bekkering, S. et al. Trained immunity: linking obesity and cardiovascular disease across the life-course? Trends Endocrinol. Metab. 31, 378-389 (2020).

79. Pink, C. et al. Longitudinal effects of systemic inflammation markers on periodontitis. J. Clin. Periodontol. 42, 988-997 (2015).

80. Wright, H. J., Matthews, J. B., Chapple, I. L., Ling-Mountford, N. \& Cooper, P. R. Periodontitis associates with a type 1 IFN signature in periphera blood neutrophils. J. Immunol. 181, 5775-5784 (2008).

81. Kalafati, L. et al. Innate immune training of granulopoiesis promotes anti-tumor activity. Cell 183 771-785 (2020).

82. Rafferty, B. et al. Impact of monocytic cells on recovery of uncultivable bacteria from atherosclerotic lesions. J. Intern. Med. 270, 273-280 (2011)

83. Kozarov, E. V., Dorn, B. R., Shelburne, C. E., Dunn, W. A. Jr \& Progulske-Fox, A. Human atherosclerotic plaque contains viable invasive Actinobacillus actinomycetemcomitans and Porphyromonas gingivalis. Arterioscler. Thromb. Vasc. Biol. 25, e17-e18 (2005).

84. Carrion, J. et al. Microbial carriage state of peripheral blood dendritic cells (DCs) in chronic periodontitis influences DC differentiation, atherogenic potential. J. Immunol. 189, 3178-3187 (2012).

85. Gevers, D. et al. The treatment-naive microbiome in new-onset Crohn's disease. Cell Host Microbe 15 382-392 (2014).

86. Schirmer, M. et al. Compositional and temporal changes in the gut microbiome of pediatric ulcerative colitis patients are linked to disease course. Cell Host Microbe 24, 600-610.e604 (2018).

Gevers et al. and Schirmer et al. demonstrate that the dominant species with increased abundance in paediatric patients with IBD are actually derived from the oral cavity instead of being typical intestinal bacteria

87. Lamont, R. J., Koo, H. \& Hajishengallis, G. The oral microbiota: dynamic communities and host interactions. Nat. Rev. Microbiol. 16, 745-759 (2018).

88. Gimbrone, M. A. Jr \& García-Cardeña, G. Endothelial cell dysfunction and the pathobiology of atherosclerosis. Circ. Res. 118, 620-636 (2016)

89. Mougeot, J. C. et al. Porphyromonas gingivalis is the most abundant species detected in coronary and femoral arteries. J. Oral Microbiol. 9, 1281562 (2017).

90. Dioguardi, M. et al. The role of periodontitis and periodontal bacteria in the onset and progression of Alzheimer's disease: a systematic review. J. Clin. Med. 9, 495 (2020).

91. Beydoun, M. A. et al. Clinical and bacterial markers of periodontitis and their association with incident all-cause and Alzheimer's disease dementia in a large national survey. J. Alzheimers Dis. 75, 157-172 (2020).

92. Kovacech, B. \& Novak, M. Tau truncation is a productive posttranslational modification of neurofibrillary degeneration in Alzheimer's disease. Curr. Alzheimer Res. 7, 708-716 (2010).

93. Long, J. M. \& Holtzman, D. M. Alzheimer disease: an update on pathobiology and treatment strategies. Cell 179, 312-339 (2019).

94. Muñoz, S. S., Garner, B. \& Ooi, L. Understanding the role of ApoE fragments in Alzheimer's disease. Neurochem. Res. 44, 1297-1305 (2019).

95. Lönn, J. et al. Lipoprotein modifications by gingipains of Porphyromonas gingivalis. J. Periodont. Res. 53, 403-413 (2018).

96. Poole, S. et al. Active invasion of Porphyromonas gingivalis and infection-induced complement activation in $\mathrm{ApoE}^{-1-}$ mice brains. J. Alzheimers Dis. 43, 67-80 (2015)

97. Hajishengallis, G., Reis, E. S., Mastellos, D. C., Ricklin, D. \& Lambris, J. D. Novel mechanisms and functions of complement. Nat. Immunol. 18 1288-1298 (2017).

98. Yin, C. et al. ApoE attenuates unresolvable inflammation by complex formation with activated C1q. Nat. Med. 25, 496-506 (2019)

99. Kantarci, A. et al. Combined administration of resolvin $\mathrm{E} 1$ and lipoxin $\mathrm{A} 4$ resolves inflammation in a murine model of Alzheimer's disease. Exp. Neurol. 300 , 111-120 (2018).

100. Kantarci, A. et al. Microglial response to experimental periodontitis in a murine model of Alzheimer's disease. Sci. Rep. 10, 18561 (2020).

101. Papageorgiou, S. N. et al. Inflammatory bowel disease and oral health: systematic review and a meta-analysis. J. Clin. Periodontol. 44, 382-393 (2017).

102. Pietropaoli, D. et al. Occurrence of spontaneous periodontal disease in the SAMP1/YitFc Murine model of Crohn disease. J. Periodontol. 85, 1799-1805 (2014).

103. Moran, C. J. et al. IL-10R polymorphisms are associated with very-early-onset ulcerative colitis. Inflamm. Bowel Dis. 19, 115-123 (2013). 
104. Schmidt, T. S. et al. Extensive transmission of microbes along the gastrointestinal tract. elife $\mathbf{8}$ e42693 (2019)

105. Yachida, S. et al. Metagenomic and metabolomic analyses reveal distinct stage-specific phenotypes of the gut microbiota in colorectal cancer. Nat. Med. 25 968-976 (2019)

106. Thomas, A. M. et al. Metagenomic analysis of colorectal cancer datasets identifies cross-cohort microbial diagnostic signatures and a link with choline degradation. Nat. Med. 25, 667-678 (2019).

107. Kostic, A. D. et al. Fusobacterium nucleatum potentiates intestinal tumorigenesis and modulates the tumor-immune microenvironment. Cell Host Microbe 14, 207-215 (2013).

108. Wirbel, J. et al. Meta-analysis of fecal metagenomes reveals global microbial signatures that are specific for colorectal cancer. Nat. Med. 25, 679-689 (2019).

109. Castellarin, M. et al. Fusobacterium nucleatum infection is prevalent in human colorectal carcinoma Genome Res. 22, 299-306 (2012).

110. Kostic, A. D. et al. Genomic analysis identifies association of Fusobacterium with colorectal carcinoma. Genome Res. 22, 292-298 (2012).

111. Figuero, E., Han, Y. W. \& Furuichi, Y. Periodontal diseases and adverse pregnancy outcomes: mechanisms. Periodontol. $200083,175-188$ (2020).

112. Rubinstein, M. R. et al. Fusobacterium nucleatum promotes colorectal carcinogenesis by modulating E-cadherin/ $\beta$-catenin signaling via its FadA adhesin. Cell Host Microbe 14, 195-206 (2013).

113. Casasanta, M. A et al. Fusobacterium nucleatum host-cell binding and invasion induces IL- 8 and CXCL1 secretion that drives colorectal cancer cell migration. Sci. Signal. 13, eaba9157 (2020).

This study identifies a new mechanism linking F. nucleatum to CRC by showing that this oral bacterium promotes chemokine secretion that stimulates tumour cell migration and invasion.

114. Yu, T. et al. Fusobacterium nucleatum promotes chemoresistance to colorectal cancer by modulating autophagy. Cell 170, 548-563 (2017).

This paper shows that $F$. nucleatum adversely affects the CRC chemotherapeutic response by modulating autophagy.

115. Komiya, Y. et al. Patients with colorectal cancer have identical strains of Fusobacterium nucleatum in their colorectal cancer and oral cavity. Gut 68, 1335-1337 (2019).

116. Abed, J. et al. Colon cancer-associated Fusobacterium nucleatum may originate from the oral cavity and reach colon tumors via the circulatory system. Front. Cell Infect. Microbiol. 10, 400 (2020).

117. Ning, Y. $\&$ Lenz, H. J. Targeting IL-8 in colorectal cancer. Expert. Opin. Ther. Targets 16, 491-497 (2012)

118. Rubinstein, M. R. et al. Fusobacterium nucleatum promotes colorectal cancer by inducing $\mathrm{Wnt} / \beta$-catenin modulator annexin A1. EMBO Rep 20, e47638 (2019).

119. Sun, J. et al. Role of the oral microbiota in cancer evolution and progression. Cancer Med. 9 6306-6321 (2020)

120. Hashimoto, M. et al. Periodontitis and Porphyromonas gingivalis in preclinical stage of arthritis patients. PLOS ONE 10, e0122121 (2015)

121. Mikuls, T. R. et al. Periodontitis and Porphyromonas gingivalis in patients with rheumatoid arthritis. Arthritis Rheumatol. 66, 1090-1100 (2014).

122. Al-Katma, M. K., Bissada, N. F., Bordeaux, J. M., Sue, J. \& Askari, A. D. Control of periodontal infection reduces the severity of active rheumatoid arthritis. J. Clin. Rheumatol. 13, 134-137 (2007).

123. Khare, N. et al. Nonsurgical periodontal therapy decreases the severity of rheumatoid arthritis: a case-control study. J. Contemp. Dent. Pract. 17 484-488 (2016).

124. Ortiz, P. et al. Periodontal therapy reduces the severity of active rheumatoid arthritis in patients treated with or without tumor necrosis factor inhibitors. J. Periodontol. 80, 535-540 (2009).

125. Maresz, K. J. et al. Porphyromonas gingivalis facilitates the development and progression of destructive arthritis through its unique bacterial peptidylarginine deiminase (PAD). PLoS Pathog. 9 , e1003627 (2013).

126. Gully, N. et al. Porphyromonas gingivalis peptidylarginine deiminase, a key contributor in the pathogenesis of experimental periodontal disease and experimental arthritis. PLOS ONE 9, e100838 (2014).

Maresz et al. is the first study to mechanistically link a periodontal pathogen ( $P$. gingivalis) with generation of citrullinated autoantigens that are involved in the pathogenesis of rheumatoid arthritis. Gully et al. provide independent confirmation that peptidylarginine deiminase of $P$. gingivalis promotes the induction of ACPAs and experimental arthritis.

127. Wegner, N. et al. Peptidylarginine deiminase from Porphyromonas gingivalis citrullinates human fibrinogen and alpha-enolase: implications for autoimmunity in rheumatoid arthritis. Arthritis Rheumatol. 62, 2662-2672 (2010).

128. Lübcke, P. M. et al. Periodontal treatment prevents arthritis in mice and methotrexate ameliorates periodontal bone loss. Sci. Rep. 9,8128 (2019).

129. Flak, M. B. et al. Inflammatory arthritis disrupts gut resolution mechanisms, promoting barrier breakdown by Porphyromonas gingivalis. JCl Insight 4, e 125191 (2019).

130. Boyaka, P. N. \& Fujihashi, K. in Clinical Immunology (Fifth Edition) (eds Rich, R. R. et al.) 285-298 (Elsevier, 2019).

131. Gill, N., Wlodarska, M. \& Finlay, B. B. The future of mucosal immunology: studying an integrated system-wide organ. Nat. Immunol. 11, 558-560 (2010).

132. Scribano, M. L. Vedolizumab for inflammatory bowe disease: from randomized controlled trials to real-life evidence. World J. Gastroenterol. 24, 2457-2467 (2018).

133. Calderón-Gómez, E. et al. Commensal-specific CD4 cells from patients with Crohn's disease have a T-helper 17 inflammatory profile. Gastroenterology 151, 489-500 (2016).

134. Wang, J. et al. Respiratory influenza virus infection induces intestinal immune injury via microbiotamediated Th1 7 cell-dependent inflammation. J. Exp. Med. 211, 2397-2410 (2014).

135. D'Amico, F., Baumgart, D. C., Danese, S. \& Peyrin-Biroulet, L. Diarrhea during COVID-19 infection: pathogenesis, epidemiology, prevention, and management. Clin. Gastroenterol. Hepatol. 18 1663-1672 (2020).

136. Miles, B. et al. Secondary lymphoid organ homing phenotype of human myeloid dendritic cells disrupted by an intracellular oral pathogen. Infect. Immun. $\mathbf{8 2}$ 101-111 (2014)

137. Sainz, J. \& Sata, M. CXCR4, a key modulator of vascular progenitor cells. Arterioscler. Thromb. Vasc. Biol. 27, 263-265 (2007).

138. Rajendran, M. et al. Systemic antibiotic therapy reduces circulating inflammatory dendritic cells and Treg-Th 17 plasticity in periodontitis. J. Immunol. 202 2690-2699 (2019).

139. Chen, M. et al. Dendritic cell apoptosis in the maintenance of immune tolerance. Science 311 1160-1164 (2006)

140. Hajishengallis, G., Chavakis, T. \& Lambris, J. D. Current understanding of periodontal disease pathogenesis and targets for host-modulation therapy. Periodontol. 2000 84, 14-34 (2020).

141. Ridker, P. M. et al. Antiinflammatory therapy with canakinumab for atherosclerotic disease. N. Engl. J. Med. 377, 1119-1131 (2017)

142. Jaiswal, S. \& Libby, P. Clonal haematopoiesis: connecting ageing and inflammation in cardiovascular disease. Nat. Rev. Cardiol. 17, 137-144 (2020).

143. Fuster, J. J. et al. Clonal hematopoiesis associated with TET2 deficiency accelerates atherosclerosis development in mice. Science 355, 842-847 (2017). This study provides causal evidence linking CHIP to atherosclerosis. The authors show that atherosclerosis progresses more rapidly in mice transplanted with TET2-deficient bone marrow cells, in great part attributed to the increased inflammatory activity of TET2-deficient macrophage progeny.
144. Eskan, M. A et al. The leukocyte integrin antagonist Del-1 inhibits IL-17-mediated inflammatory bone loss. Nat. Immunol. 13, 465-473 (2012).

145. Shin, J. et al. DEL-1 restrains osteoclastogenesis and inhibits inflammatory bone loss in nonhuman primates. Sci. Transl. Med. 7, 307ra155 (2015).

146. Kourtzelis, I. et al. DEL-1 promotes macrophage efferocytosis and clearance of inflammation. Nat. Immunol. 20, 40-49 (2019).

147. Li, X. et al. The DEL-1/ß3 integrin axis promotes regulatory $\mathrm{T}$ cell responses during inflammation resolution. J. Clin. Invest. 130, 6261-6277 (2020).

148. Mitroulis, I. et al. Secreted protein Del-1 regulates myelopoiesis in the hematopoietic stem cell niche. J. Clin. Invest. 127, 3624-3639 (2017).

149. Adler, C. J. et al. Sequencing ancient calcified dental plaque shows changes in oral microbiota with dietary shifts of the Neolithic and Industrial revolutions. Nat. Genet. 45, 450-455 (2013).

150. Peres, M. A. et al. Oral diseases: a global public health challenge. Lancet 394, 249-260 (2019).

151. Dutzan, N. et al. A dysbiotic microbiome triggers TH1 7 cells to mediate oral mucosal immunopathology in mice and humans. Sci. Transl. Med. 10, eaat0797 (2018)

152. Abe, T. $\&$ Hajishengallis, G. Optimization of the ligature-induced periodontitis model in mice. J. Immunol. Methods 394, 49-54 (2013).

153. Hajishengallis, G. et al. Low-abundance biofilm species orchestrates inflammatory periodontal disease through the commensal microbiota and complement. Cell Host Microbe 10, 497-506 (2011).

154. Payne, M. A. et al. Horizontal and vertical transfer of oral microbial dysbiosis and periodontal disease. J. Dent. Res. 98, 1503-1510 (2019).

155. Penkov, S., Mitroulis, I., Hajishengallis, G. \& Chavakis, T. Immunometabolic crosstalk: an ancestral principle of trained immunity? Trends Immunol. 40, 1-11 (2019).

156. Kaufmann, E. et al. BCG educates hematopoietic stem cells to generate protective innate immunity against tuberculosis. Cell 172, 176-190 (2018).

157. Nahrendorf, M. Myeloid cell contributions to cardiovascular health and disease. Nat. Med. 24 711-720 (2018)

158. Murphy, A. J. \& Tall, A. R. Disordered haematopoiesis and athero-thrombosis. Eur. Heart J. 37, 1113-1121 (2016).

159. Barrett, T. J., Murphy, A. J., Goldberg, I. J. \& Fisher, E. A. Diabetes-mediated myelopoiesis and the relationship to cardiovascular risk. Ann. N. Y Acad. Sci. 1402, 31-42 (2017).

160. Tall, A. R. \& Yvan-Charvet, L. Cholesterol, inflammation and innate immunity. Nat. Rev. Immunol. 15, 104-116 (2015)

Acknowledgements

The authors' research is supported by grants from the US National Institutes of Health (DE024153, DE024716, DE029436 to G.H.; DE026152 and DE028561 to G.H. and TC.) and the German Research Foundation (CRC-TR127 and CRC 1181 to T.C.). The authors regret that several important studies could only be cited indirectly through comprehensive reviews, owing to space and reference number imitations.

Author contributions

The authors contributed equally to all aspects of the article.

Competing interests

The authors declare no competing interests.

Peer review information

Nature Reviews Immunology thanks N. Kamada, T. Van Dyke and the other, anonymous, reviewer(s) for their contribution to the peer review of this work.

\section{Publisher's note}

Springer Nature remains neutral with regard to jurisdictional claims in published maps and institutional affiliations.

(c) Springer Nature Limited 2021 